\title{
Condensational and Collisional Growth of Cloud Droplets in a Turbulent Environment
}

\author{
XiAng-Yu Li, ${ }^{\mathrm{a}, \mathrm{b}, \mathrm{c}, \mathrm{d}, \mathrm{e}, \mathrm{f}}$ AXEl BrandenburG,${ }^{\mathrm{c}, \mathrm{e}, \mathrm{f}, \mathrm{g}}$ Gunilla SvensSOn,${ }^{\mathrm{a}, \mathrm{b}, \mathrm{d}}$ Nils E. L. Haugen, ${ }^{\mathrm{h}, \mathrm{i}}$ \\ BERNHARD MEHLIG, ${ }^{\mathrm{j}}$ AND IGOR ROGACHEVSKII ${ }^{\mathrm{c}, \mathrm{k}}$ \\ ${ }^{a}$ Department of Meteorology, Stockholm University, Stockholm, Sweden \\ ${ }^{\mathrm{b}}$ Bolin Centre for Climate Research, Stockholm University, Stockholm, Sweden \\ ${ }^{\mathrm{c}}$ Nordita, KTH Royal Institute of Technology and Stockholm University, Stockholm, Sweden \\ ${ }^{\mathrm{d}}$ Swedish e-Science Research Centre, Stockholm, Sweden \\ ${ }^{\mathrm{e}}$ Joint Institute for Laboratory Astrophysics, University of Colorado Boulder, Boulder, Colorado \\ ${ }^{\mathrm{f}}$ Laboratory for Atmospheric and Space Physics, University of Colorado Boulder, Boulder, Colorado \\ ${ }^{\mathrm{g}}$ Department of Astronomy, Stockholm University, Stockholm, Sweden \\ ${ }^{\mathrm{h}}$ SINTEF Energy Research, Trondheim, Norway \\ ${ }^{\mathrm{i}}$ Department of Energy and Process Engineering, Norwegian University of Science and Technology (NTNU), \\ Trondheim, Norway \\ ${ }^{\mathrm{j}}$ Department of Physics, Gothenburg University, Gothenburg, Sweden \\ ${ }^{\mathrm{k}}$ Department of Mechanical Engineering, Ben-Gurion University of the Negev, Beer-Sheva, Israel
}

(Manuscript received 24 April 2019, in final form 29 September 2019)

\begin{abstract}
We investigate the effect of turbulence on the combined condensational and collisional growth of cloud droplets by means of high-resolution direct numerical simulations of turbulence and a superparticle approximation for droplet dynamics and collisions. The droplets are subject to turbulence as well as gravity, and their collision and coalescence efficiencies are taken to be unity. We solve the thermodynamic equations governing temperature, water vapor mixing ratio, and the resulting supersaturation fields together with the Navier-Stokes equation. We find that the droplet size distribution broadens with increasing Reynolds number and/or mean energy dissipation rate. Turbulence affects the condensational growth directly through supersaturation fluctuations, and it influences collisional growth indirectly through condensation. Our simulations show for the first time that, in the absence of the mean updraft cooling, supersaturation-fluctuation-induced broadening of droplet size distributions enhances the collisional growth. This is contrary to classical (nonturbulent) condensational growth, which leads to a growing mean droplet size, but a narrower droplet size distribution. Our findings, instead, show that condensational growth facilitates collisional growth by broadening the size distribution in the tails at an early stage of rain formation. With increasing Reynolds numbers, evaporation becomes stronger. This counteracts the broadening effect due to condensation at late stages of rain formation. Our conclusions are consistent with results of laboratory experiments and field observations, and show that supersaturation fluctuations are important for precipitation.
\end{abstract}

\section{Introduction}

It has been suggested that warm rain accounts for about $30 \%$ of the total amount of rain and for $70 \%$ of the total rain area in the tropics, which plays an important role in regulating the vertical water and energy transport of the tropical atmosphere (Lau and $\mathrm{Wu} 2003$ ). Its rapid formation has puzzled the cloud microphysics community for about 70 years. The observed time scale of warm rain formation is known to be about $20 \mathrm{~min}$ (Stephens and Haynes 2007), which is much shorter than the theoretically

Corresponding author: Xiang-Yu Li, xiang.yu.li@su.se predicted time scale of $8 \mathrm{~h}$ (Saffman and Turner 1956) and $60 \mathrm{~min}$ in simulations of classical adiabatic parcel models (Jonas 1996). Condensational and collisional growth determine the formation of warm rain. In the absence of turbulence, condensational growth is effective for cloud condensation nuclei and cloud droplets smaller than $15 \mu \mathrm{m}$ in radius. Since the growth rate is inversely proportional to the radius, condensational growth leads to a narrow width of the droplet size distribution. The gravity-generated collisional growth in isolation becomes important only when the mean radius of droplets is larger than $\sim 40 \mu \mathrm{m}$, and the collision efficiency becomes large enough for collisional 
growth. Thus, there is a size gap of $15-40 \mu \mathrm{m}$ where neither condensation nor collision-coalescence drives the growth (Pruppacher and Klett 2012; Lamb and Verlinde 2011; Grabowski and Wang 2013). Therefore, the effect of turbulence on condensational and collisional growth has been proposed to overcome this size gap (Saffman and Turner 1956; Shaw 2003; Khain et al. 2007; Devenish et al. 2012; Grabowski and Wang 2013). In the meteorology community, the process of collision-coalescence is also referred to as collection (Berry and Reinhardt 1974), while in the astrophysical community, this process is referred to as coagulation (Li et al. 2018a; Johansen and Lambrechts 2017). Since we assume unit collision and coalescence efficiency, we use the terminology collision in the present study.

Saffman and Turner (1956) showed that turbulent mixing enhances the droplet collision rate, following an idea of Smoluchowsky (1917). They found that this rate is proportional to the mean energy dissipation rate of the turbulent flow. The calculation assumes that the droplets are so small (about $10 \mu \mathrm{m}$ in radius) that inertial effects [see Gustavsson and Mehlig (2016) for a review] are negligible. More recently it has become clear that inertial effects can significantly increase the collision rate for larger droplets, with larger Stokes numbers (Sundaram and Collins 1997; Falkovich et al. 2002; Chun et al. 2005; Wilkinson et al. 2006; Salazar et al. 2008; Bec et al. 2010; Gustavsson and Mehlig 2011, 2014; Gustavsson et al. 2014; Meibohm et al. 2017). These predictions are in good agreement with direct numerical simulations (DNS) of droplet dynamics in turbulence (Bhatnagar et al. 2018a,b), but the effect applies only to droplets that are large enough that they can frequently detach from the flow, due to the formation of caustics (Wilkinson and Mehlig 2005). This requires Stokes numbers of order unity.

Reuter et al. (1988), Grover and Pruppacher (1985), Pinsky and Khain (2004), and Pinsky et al. (2007, 2008) also suggested that turbulence may cause a substantial enhancement of the collision rate, yet Koziol and Leighton (1996) found that turbulence only has a moderate effect on the collision rate. This may partially be due to small Stokes numbers.

Recently it has become feasible to study the condensational and collisional growth using DNS. Most DNS studies of droplet collisions in turbulence (Franklin et al. 2005; Ayala et al. 2008; Rosa et al. 2013; Chen et al. 2016; Woittiez et al. 2009) record collision frequencies but do not allow the droplets to coalesce and grow. It is then not possible to study how the droplet size distribution develops. Nevertheless, those works revealed that turbulence enhances the collision rate, and the effect is larger for larger mean energy dissipation rates. The value of the Reynolds number, by contrast, was found to be of secondary importance.

Franklin (2008), Xue et al. (2008), and Wang and Grabowski (2009) investigated the collision-coalescence processes by solving the Smoluchowski equation together with the Navier-Stokes equation using DNS. They found that the size distribution of cloud droplets is significantly enhanced by turbulence. Onishi and Seifert (2016) extended the collision-rate model of Wang and Grabowski (2009) and performed DNS at higher Reynolds number, where a Reynolds number dependency was obtained. Using a Lagrangian collision-detection method, Chen et al. (2018a) found that turbulence strongly affects the broadening of the size distribution. Li et al. (2018a) showed that, in the absence of condensation, turbulence enhances the collision-coalescence process. They also found that this enhancement effect is sensitive to the initial width of the droplet size distribution.

The effect of turbulence on condensational growth has been explored intensively. Since turbulence affects the temperature field and spatial distribution of the water vapor mixing ratio, the supersaturation field determined by temperature and water mixing ratio is inevitably affected by turbulence. Srivastava (1989) criticized the use of volume-averaged supersaturation and proposed adopting the local supersaturation field to calculate the condensational growth of cloud droplets. This is a prototype of supersaturation fluctuations. To investigate how local supersaturation fluctuations affect the condensational droplet growth in the cloud core, Vaillancourt et al. (2002) solved the thermodynamical equations that govern the supersaturation using DNS in the presence of a turbulent flow, taking into account the mean updraft cooling, gravitational settling, droplet inertia, and latent heat release. Vaillancourt et al. (2002) concluded that the width of the droplet size distribution decreases as the turbulent mean energy dissipation rate increases and attributed this to the decrease in the decorrelation time of the supersaturation fluctuation. Lanotte et al. (2009), Sardina et al. (2015), and Siewert et al. (2017) performed DNS for condensational growth using a slightly simpler model that accounts for supersaturation fluctuations but not for details of the thermodynamics. They found that the size distribution broadens as the Reynolds number increases. Paoli and Shariff (2009) found that the entrainment-induced supersaturation fluctuations broaden the droplet size distribution. Their study is based on stochastically forced temperature and vapor fields. Grabowski and Abade (2017) and Abade et al. (2018) came to a similar conclusion using a turbulent adiabatic-parcel model. Li et al. (2019) confirmed that the droplet size distribution broadens with increasing Reynolds number and is 
insensitive to the mean energy dissipation rate of turbulence. Field observations of the supersaturation fluctuations and the droplet-size distribution (Siebert and Shaw 2017; Yang et al. 2018; Desai et al. 2019) also support the idea that supersaturation fluctuations due to turbulence lead to broadening of droplet size distribution.

Most of the previous DNS studies only considered either condensational growth or collisional growth. The combined condensational and collisional growth has rarely been investigated. Recently, Saito and Gotoh (2018) studied the combined processes using DNS. They found that the width of the droplet size distribution increases as the turbulence intensity increases. However, they did not discuss whether it is the Reynolds number or the mean energy dissipation rate that matters for the broadening. Chen et al. (2018b) employed the same model as Saito and Gotoh (2018) and concluded that droplet size distributions broaden with increasing mean energy dissipation rate. However, they did not study the dependency of the droplet size distribution upon the Reynolds number. Indeed, several works (Lanotte et al. 2009; Sardina et al. 2015; Siewert et al. 2017; Li et al. 2019) suggested that condensational growth is sensitive to the Reynolds number. Collisional growth, however, is mainly affected by the mean energy dissipation rate (Ayala et al. 2008; Chen et al. 2016; Li et al. 2018a).

In this paper, we investigate the effect of turbulence on the combined condensational and collisional growth of cloud droplets at high Reynolds numbers using DNS of turbulence. We strive to investigate whether/how supersaturationfluctuation-induced broadening of droplet size distributions affect the collision-coalescence process, and thereby the warm rain formation. For the dynamics of the local temperature and the local water vapor mixing ratio we use the same model as Vaillancourt et al. (2002), Saito and Gotoh (2018), and Chen et al. (2018b), excluding the mean updraft cooling (see below). Details of our implementation are given in Li et al. (2019). The droplet dynamics in our simulations is coupled to the turbulence through Stokes force. The droplets are also subject to gravitational settling. DNS of the combined problem poses formidable challenges. Therefore we use a stochastic Monte Carlo approximation, the superparticle method (Zsom and Dullemond 2008; Shima et al. 2009; Li et al. 2017; Unterstrasser et al. 2017), for the collision-coalescence process. Strengths and weaknesses of the method were discussed by Li et al. (2018b). Since we focus on the impact of turbulence on droplet growth, we omit the effect of cooling due to a mean updraft.

We first investigate how condensational and collisional processes affect each other through thermodynamics and droplet dynamics. Second, we explore how the combined condensational and collisional droplet growth depends on the mean energy dissipation rate and upon the Reynolds number. We focus on the droplet size distribution, which is the key to cloud-climate feedback and precipitation (Shaw 2003). We show that collisional growth is enhanced by the appearance of a broadening tail of the droplet size distribution through supersaturation fluctuations.

\section{Numerical model}

The equations governing the Eulerian fields and condensation are the same as the standard ones (Vaillancourt et al. 2002), and their implementation is described in Li et al. (2019). For the collision-coalescence dynamics we use the superparticle method, which has been validated in $\mathrm{Li}$ et al. (2017). The Pencil code is used for all the simulations.

\section{a. Eulerian fields and condensation}

We use the standard equations for fluid density $\rho(\mathbf{x}, t)$, fluid velocity $\mathbf{u}(\mathbf{x}, t)$, temperature $T(\mathbf{x}, t)$, and water vapor mixing ratio $q_{v}(\mathbf{x}, t)$ :

$$
\begin{aligned}
& \frac{\partial \rho}{\partial t}+\nabla \cdot(\rho \mathbf{u})=\mathbf{S}_{\rho} \\
& \frac{D \mathbf{u}}{D t}=\mathbf{F}-\rho^{-1} \nabla p+\rho^{-1} \nabla \cdot(2 \nu \rho \mathbf{S})+B \mathbf{e}_{z}+\mathbf{S}_{u} \\
& \frac{D T}{D t}=\kappa \nabla^{2} T+\frac{L}{c_{p}} C_{d} \\
& \frac{D q_{v}}{D t}=D \nabla^{2} q_{v}-C_{d}
\end{aligned}
$$

where turbulence is driven by a stochastic forcing function $\mathbf{F}$ [see Haugen et al. (2004) for details], $D / D t=$ $\partial / \partial t+\mathbf{u} \cdot \nabla$ denotes the advective derivative, and $\mathbf{S}_{i j}=$ $\left[(1 / 2)\left(\partial_{j} u_{i}+\partial_{i} u_{j}\right)\right]-(1 / 3) \delta_{i j} \nabla \cdot \mathbf{u}$ is the rate-of-strain tensor (subtracting the divergence makes it traceless), $p$ and $\rho$ are gas pressure and density, and $L$ is the latent heat. The parameters $D$ and $\kappa$ are the diffusivities of water vapor and temperature. The source terms $\mathbf{S}_{\rho}$ and $\mathbf{S}_{u}$ in Eqs. (1) and (2) describe mass transfer between the droplets and the humid air due to condensation and evaporation. In our case, the mass transfer is small relative to the total air mass, and the fraction of liquid to gaseous mass is also low. Therefore, we neglect these terms. The pressure $p$ and the density $\rho$ are related to each other by an adiabatic equation of state, $p=\rho c_{s}^{2} / \gamma$, where $c_{s}=5.0 \mathrm{~m} \mathrm{~s}^{-1}$ is the sound speed set in the code, $\gamma=c_{p} / c_{v}=7 / 5$, with $c_{p}=1005 \mathrm{~J} \mathrm{~kg}^{-1} \mathrm{~K}^{-1}$ being the specific heat at constant pressure and $c_{v}$ the specific heat at constant volume. For the kinematic viscosity and the thermal diffusivity of air, we use $\nu=\kappa=1.5 \times 10^{-5} \mathrm{~m}^{2} \mathrm{~s}^{-1}$. 
Furthermore, $D=2.55 \times 10^{-5} \mathrm{~m}^{2} \mathrm{~s}^{-1}$ is the water vapor diffusivity and $L=2.5 \times 10^{6} \mathrm{~J} \mathrm{~kg}^{-1}$ is the latent heat.

The buoyancy force $B(\mathbf{x}, t)$ is determined by the temperature $T(\mathbf{x}, t)$ through $B=g\left(T^{\prime} / T+\alpha q_{v}^{\prime}-q_{l}\right)$, where $g=9.81 \mathrm{~m} \mathrm{~s}^{-2}$ is the gravitational acceleration, $T^{\prime}=T-T_{\text {env }}$ is the temperature fluctuation with respect to the environmental temperature $T_{\text {env }}=293 \mathrm{~K}, \alpha=0.608$ is the expansion coefficient, and $q_{v}^{\prime}=q_{v}-q_{v \text {,env }}$ is the fluctuation of the water vapor mixing ratio (Lamb and Verlinde 2011; Kumar et al. 2014), with $q_{v \text {,env }}=0.01 \mathrm{~kg} \mathrm{~kg}^{-1}$; see also Li et al. (2019). This follows the common approach (Vaillancourt et al. 2002) in that it uses the Boussinesq approximation to describe the term $B \mathbf{e}_{z}\left(\mathbf{e}_{z}\right.$ is the unit vector in the vertical direction) in Eq. (2), assuming that density variations are negligible except when multiplied by the gravitational acceleration; see, for example, Mehaddi et al. (2018). This requires that temperature gradients are small. Our implementation is slightly different from the classical Boussinesq approximation, where $\nabla \cdot \mathbf{u}=0$ is assumed. Here, we use instead the full continuity equation [Eq. (1)].

Both $T$ and $q_{v}$ are affected by droplets via the condensation rate $C_{d}$ (Vaillancourt et al. 2001; Li et al. 2019):

$$
C_{d}(\mathbf{x}, t)=\frac{4 \pi \rho_{l} G}{\rho_{a}}\langle s(\mathbf{x}, t) r(t)\rangle \bar{n} .
$$

The average $\langle\cdots\rangle$ represents a local average over droplets at position $\mathbf{x}$ and of volume $\eta^{3}$, where $\eta$ is the Kolmogorov length, and $\bar{n}=N_{\Delta} /(\Delta x)^{3}$ is the number of droplets $N_{\Delta}$ per grid volume $(\Delta x)^{3}$. The parameters are liquid-water density $\rho_{l}=1000 \mathrm{~kg} \mathrm{~m}^{-3}$, reference mass density of dry air $\rho_{a}=1 \mathrm{~kg} \mathrm{~m}^{-3}$, condensation parameter $G=1.17 \times 10^{-10} \mathrm{~m}^{2} \mathrm{~s}^{-1}$, supersaturation $s(\mathbf{x}, t)=q_{v} / q_{v s}(T)-1$, and saturated water vapor mixing ratio $q_{v s}(T)=e_{s}(T) / R_{v} \rho_{0} T$ with gas constant $R_{v}=461.5 \mathrm{~J} \mathrm{~kg}^{-1} \mathrm{~K}^{-1}$. Finally, $e_{s}$ is the saturation pressure obtained from the Clausius-Clapeyron equation (Yau and Rogers 1996; Götzfried et al. 2017): $e_{s}(T)=$ $c_{1} \exp \left(-c_{2} / T\right)$. For the two constants, we choose $c_{1}=$ $2.53 \times 10^{11} \mathrm{~Pa}$ and $c_{2}=5420 \mathrm{~K}$, as in Li et al. (2019).

\section{b. Droplet dynamics and collisions: The superparticle algorithm}

We approximate the droplet dynamics using the superparticle method (Zsom and Dullemond 2008; Shima et al. 2009; Johansen et al. 2012; Li et al. 2017, 2018a). In this approach, several identical microscopic droplets are represented by a superparticle. Each superparticle is assumed a certain volume and is thus assigned a droplet number density, $n_{i}$. The position of superparticle $i$ is denoted by $\mathbf{x}_{i}$ and obeys

$$
\frac{d \mathbf{x}_{i}}{d t}=\mathbf{V}_{i}
$$

where $\mathbf{V}_{i}$ is the velocity of the superparticle. The acceleration obeys Stokes law,

$$
\frac{d \mathbf{V}_{i}}{d t}=\frac{1}{\tau_{i}}\left(\mathbf{u}-\mathbf{V}_{i}\right)+\mathbf{g}
$$

where $\tau_{i}$ is the Stokes time, $\mathbf{u}$ is the fluid velocity at $\mathbf{x}_{i}$, and $\mathbf{g}$ is the gravitational acceleration. The value of $\tau_{i}$ of superparticle $i$ is given by

$$
\tau_{i}=2 \rho_{d} r_{i}^{2} /\left[9 \rho \nu\left(1+0.15 \operatorname{Re}_{i}^{2 / 3}\right)\right]
$$

Here the term $1+0.15 \operatorname{Re}_{i}^{2 / 3}$ (Schiller and Naumann 1933; Marchioli et al. 2008) is due to particle Reynolds numbers, $\operatorname{Re}_{i}=2 r_{i}\left|\mathbf{u}-\mathbf{V}_{i}\right| / \nu$. We adopt this term since the maximum particle Reynolds number becomes large when $r$ exceeds values of around $r=100 \mu \mathrm{m}$, and the linear Stokes drag does not hold. Droplet collisions are modeled as follows (Shima et al. 2009; Johansen et al. 2012; Li et al. 2017; Unterstrasser et al. 2017). When two superparticles reside in the same grid cell, the probability of collision between one droplet in a superparticle with a droplet in another superparticle during time step $\Delta t$ is $p_{c}=\tau_{c}^{-1} \Delta t$. The collision time $\tau_{c}$ is determined by

$$
\tau_{c}^{-1}=\sigma_{c} n_{j}\left|\mathbf{V}_{i}-\mathbf{V}_{j}\right| E_{c} .
$$

Here $\sigma_{c}=\pi\left(r_{i}+r_{j}\right)^{2}$ is the cross section between two colliding droplets. The collision efficiency $E_{c}$ is treated as unity. We refer to Li et al. (2017, 2018a) for details of the algorithm.

The cloud system is very dilute, with a typical mean number density of about $10^{8} \mathrm{~m}^{-3}$ in stratocumulus clouds. Considering a $1 \mathrm{~m}$ cubic domain in the cloud core, the number of droplets is $10^{8}$. The typical Kolmogorov length scale is about $\eta=1 \mathrm{~mm}$. To resolve the Kolmogorov scale of cloud-like turbulence in clouds, about $(1 \mathrm{~m} / 1 \mathrm{~mm})^{3}=10^{9}$ grid points are needed in DNS. This means that there is only 1 cloud droplet in a cube with volume $(10 \eta)^{3}$, that is, 1 droplet in every 10 grid boxes in DNS. With such a dilute system, stochasticity is argued to become important for the collision-coalescence process (Kostinski and Shaw 2005; Wilkinson 2016). The inherent stochastic property of the superparticle approach renders it an ideal method to study the collision- coalescence process in cloud system (Dziekan and Pawlowska 2017; Unterstrasser et al. 2017; Grabowski et al. 2018). This realization emerged as an important consensus among Shima, Unterstrasser, Dziekan, and others 
TABLE 1. Parameter values used in the different simulation runs. "Cond" refers to condensation, "Coll" refers to collision, and "Both" refers to combined condensation and collision. Runs C1 and E1 are reference runs that agree with runs A and C of Li et al. (2019) (condensation only) and runs C2 and E2 are similar to runs A and C of Li et al. (2018a) (collisions only, except that here the initial mean number density of droplets is $n_{0}=2.5 \times 10^{8} \mathrm{~m}^{-3}$ ). To allow for a comparison with the reference runs, we chose the parameters for runs A, $\mathrm{B}, \mathrm{C}, \mathrm{D}$, and $\mathrm{E}$ to be the same as those by Li et al. (2019). These authors studied only condensation. Here collisions and condensation are treated together. The amplitude of the random forcing $F_{0}$, the lateral size of the cubic simulation box $L_{x}$, the number of grid cells $N_{\text {grid }}$, and the eddy turnover time $\tau_{L}$ were defined in section $2 \mathrm{c}$.

\begin{tabular}{|c|c|c|c|c|c|c|c|c|c|c|c|}
\hline Run & $F_{0}$ & $L_{x}(\mathrm{~m})$ & $N_{\text {grid }}$ & $N_{s}$ & Processes & $u_{\mathrm{rms}}\left(\mathrm{m} \mathrm{s}^{-1}\right)$ & $\mathrm{Re}_{\lambda}$ & $\bar{\varepsilon}\left(\mathrm{m}^{2} \mathrm{~s}^{-3}\right)$ & $\eta(\mathrm{mm})$ & $\tau_{\eta}(\mathrm{s})$ & $\tau_{L}(\mathrm{~s})$ \\
\hline A & 0.007 & 0.200 & $128^{3}$ & 244140 & Both & 0.10 & 44 & 0.005 & 0.92 & 0.056 & 0.67 \\
\hline B & 0.014 & 0.150 & $128^{3}$ & 244140 & Both & 0.14 & 45 & 0.019 & 0.65 & 0.028 & 0.35 \\
\hline $\mathrm{C}$ & 0.020 & 0.125 & $128^{3}$ & 244140 & Both & 0.16 & 45 & 0.039 & 0.54 & 0.020 & 0.25 \\
\hline $\mathrm{C} 1$ & 0.020 & 0.125 & $128^{3}$ & 244140 & Cond & 0.16 & 45 & 0.039 & 0.54 & 0.020 & 0.25 \\
\hline $\mathrm{C} 2$ & 0.020 & 0.125 & $128^{3}$ & 244140 & Coll & 0.16 & 45 & 0.039 & 0.54 & 0.020 & 0.25 \\
\hline $\mathrm{D}$ & 0.020 & 0.250 & $256^{3}$ & 1953120 & Both & 0.22 & 78 & 0.039 & 0.54 & 0.020 & 0.37 \\
\hline $\mathrm{E}$ & 0.020 & 0.500 & $512^{3}$ & 15624960 & Both & 0.28 & 130 & 0.039 & 0.54 & 0.020 & 0.58 \\
\hline E1 & 0.020 & 0.500 & $512^{3}$ & 15624960 & Cond & 0.28 & 130 & 0.039 & 0.54 & 0.020 & 0.58 \\
\hline $\mathrm{E} 2$ & 0.020 & 0.500 & $512^{3}$ & 15624960 & Coll & 0.28 & 130 & 0.039 & 0.54 & 0.020 & 0.58 \\
\hline
\end{tabular}

during a recent workshop" ${ }^{1}$ on "Eulerian vs Lagrangian methods for cloud microphysics," held in Krakow in April 2019. Comparing with the direct Lagrangian collisioncoalescence detection method, the superparticle method is computationally more efficient because it avoids having to follow each droplet individually (Shima et al. 2009; Johansen et al. 2012; Li et al. 2017).

The superparticle approach has been validated against the Smoluchowski equation in both pure gravity cases (Shima et al. 2009; Unterstrasser et al. 2017; Li et al. 2017) and in turbulent cases ( $\mathrm{Li}$ et al. 2017). Good agreement was observed. Its stochasticity was investigated by Dziekan and Pawlowska (2017), who found that the superparticle approach reproduces stochastic coalescence when $N_{p} / N_{s} \leq 9$, where $N_{p}$ is the total number of physical particles and $N_{s}$ is the total number of superparticles. This suggests that the superparticle approach does indeed capture the stochasticity of the Lagrangian collisioncoalescence detection method sufficiently accurately when $N_{p} / N_{s}$ becomes sufficiently small. Comparison with the direct Lagrangian collision-coalescence detection method is still under investigation in the cloud microphysics community. Nevertheless, Onishi et al. (2015) compared the direct collision-coalescence detection method with the Smoluchowski equation and found excellent agreement. This suggests that the superparticle approach converges to the direct Lagrangian collisioncoalescence detection method.

\section{c. DNS}

The present study builds upon our earlier simulations of condensational growth ( $\mathrm{Li}$ et al. 2019) and collisional growth (Li et al. 2018a). Here we treat both processes

\footnotetext{
${ }^{1}$ http://ww2.ii.uj.edu.pl/ arabas/workshop_2019/.
}

together in order to determine how the two mechanisms interact. Our numerical setup is the same as in Li et al. (2019), apart from the fact that we now include collisional growth. Details of our DNS solver are given in Li et al. (2017, 2019). To mimic the diluteness of the cloud system, $N_{s} / N_{\text {grid }}=0.1$ is adopted, which is within the convergence range $N_{s} / N_{\text {grid }} \leq 0.05$ ( $\mathrm{Li}$ et al. 2018a). This also ensures that the tails of $f(r, t)$ are statistically converged for larger values of $N_{s}$, and thus larger $\mathrm{Re}_{\lambda}$. More importantly, we keep $N_{p} / N_{s}=2$ so that the stochasticity of the superparticle approach is correctly represented, which is well within the limit $N_{p} / N_{s} \leq 9$ determined by Dziekan and Pawlowska (2017). Lognormal initial distributions with different width $(\sigma=0,0.02,0.05$, and 0.1$)$ are employed in the present study.

To investigate how the time evolution of droplet size distribution depends on the Taylor microscale Reynolds number $\left[\operatorname{Re}_{\lambda} \equiv u_{\mathrm{rms}}^{2} \sqrt{5 /(3 \nu \bar{\varepsilon})}\right]$ and the mean energy dissipation rate $\bar{\varepsilon}$, we performed high-resolution simulations with different domain sizes $L_{x}$ and different nondimensional forcing amplitude $F_{0}$, which is a prefactor in each Fourier component of wavevector $\mathbf{k}$ given by $F_{0} c_{s}\left(|\mathbf{k}| c_{s} / \Delta t\right)^{1 / 2}$. We choose $\mathbf{k}$ from a narrow band of wavevectors such that $|\mathbf{k}| L_{x} / 2 \pi \approx 3$.

Our results are summarized in Table 1. To elucidate the combined effect of condensational and collisional growth, we use our earlier simulations as references; see $\mathrm{Li}$ et al. (2019) for condensational growth and Li et al. (2018a) for collisional growth. The corresponding runs are also listed in the table.

We run simulations for $10 \mathrm{~min}$ even for the largest Reynolds number (runs E, E1, and E2 with $\mathrm{Re}_{\lambda}=130$ ). There are 1066600 time steps with $d t=3.405 \times 10^{-5} \mathrm{~s}$ integrated in a wall-clock time of $24 \times 11 \mathrm{~h}$ using 4096 CPUs. This corresponds to 1034 eddy turnover times. 

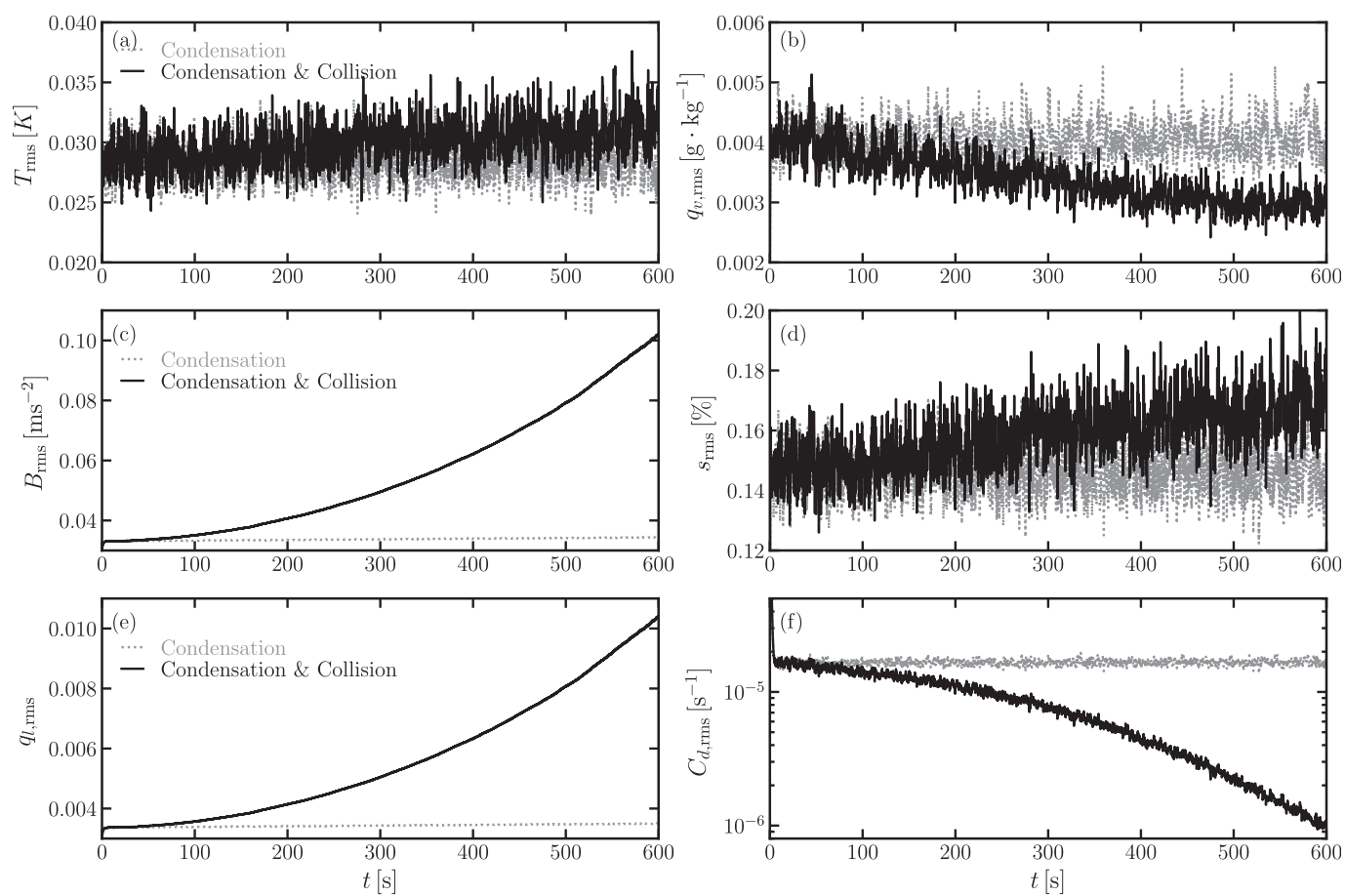

FIG. 1. Comparison of rms values of various thermodynamic quantities in the presence (absence) of collisions shown as solid (dotted) lines, corresponding to run C (C1). Condensation is included in both cases. (a) $T_{\text {rms }}(t)$, (b) $q_{v, \mathrm{rms}}(t),(\mathrm{c}) B_{\mathrm{rms}}(t),(\mathrm{d}) s_{\mathrm{rms}}(t),(\mathrm{e}) q_{l, \mathrm{rms}}(t)$, and (f) $C_{d, \mathrm{rms}}(t)$.

The droplet Stokes time is about $1.5 \times 10^{-3} \mathrm{~s}$ for the smallest droplet. Therefore, the time is well resolved.

\section{d. Diagnostics}

The tail of droplet size distribution determines warm rain formation and precipitation. We characterize the length of the tail of $f(r, t)$ by the normalized moments of $r$ as (Li et al. 2017)

$$
a_{\zeta}=\left(M_{\zeta} / M_{0}\right)^{1 / \zeta}
$$

where $M_{\zeta}=\int_{0}^{\infty} f r^{\zeta} d r$ is the $\zeta$ th moment of $r$. The case of $\zeta \rightarrow \infty$ corresponds to the maximum of $r$ and the case $\zeta=1$ corresponds to the mean radius $\bar{r}$. In practice, we choose $\zeta=24$ as a reasonably stable compromise to quantify the end of the tail of the distribution.

The relative dispersion of $f(r, t)$ is characterized by $\sigma_{r} / \bar{r}$, where $\sigma_{r}$ is the standard deviation of the droplet size and $\bar{r}$ is the mean radius (Igel and van den Heever 2017). The standard deviation of $f(r, t)$ is given by

$$
\sigma_{r}=\sqrt{a_{2}^{2}-a_{1}^{2}}
$$

in terms of the normalized moments of $r$, defined in Eq. (10). Thus $\sigma_{r} / \bar{r}=\left(a_{2}^{2}-a_{1}^{2}\right)^{1 / 2} / a_{1}$.

\section{Results}

\section{a. Comparison between cases with condensational growth, collisional growth, and with both}

Condensational growth of cloud droplets is affected by supersaturation fluctuations (Lanotte et al. 2009; Sardina et al. 2015; Siewert et al. 2017; Grabowski and Abade 2017; Li et al. 2019; Abade et al. 2018). These fluctuations are governed by temperature $T(\mathbf{x}, t)$ and by the water vapor mixing ratio $q_{v}(\mathbf{x}, t)$. We first investigate how collision impacts these quantities and therefore the condensational growth. Figure 1 shows the time series of $T_{\mathrm{rms}}(t), q_{v, \mathrm{rms}}(t), B_{\mathrm{rms}}(t), s_{\mathrm{rms}}(t), q_{l, \mathrm{rms}}(t)$, and $C_{d, \mathrm{rms}}(t)$ with and without collisions. We see that the collisioncoalescence process affects the fluctuations of these quantities to different degrees. Both $T_{\mathrm{rms}}(t)$ and $s_{\mathrm{rms}}(t)$ increase due to the collision-coalescence process while $q_{v, \text { rms }}(t)$ decreases slightly at the late stage of rain formation. This can be explained by the response of $C_{d, \mathrm{rms}}(t)$ to the collision-coalescence process. After about $t=100 \mathrm{~s}$, the collision-coalescence process becomes dominant. Since $s_{\text {rms }}(t)$ only increases slightly, $C_{d, \text { rms }}(t)$ is determined by $M_{1}$, as shown in Eq. (5). Figure 2 shows that $M_{1}$ decays rapidly as collisioncoalescence becomes important. This results in a decrease of $C_{d, \text { rms }}(t)$ by about an order of magnitude. 


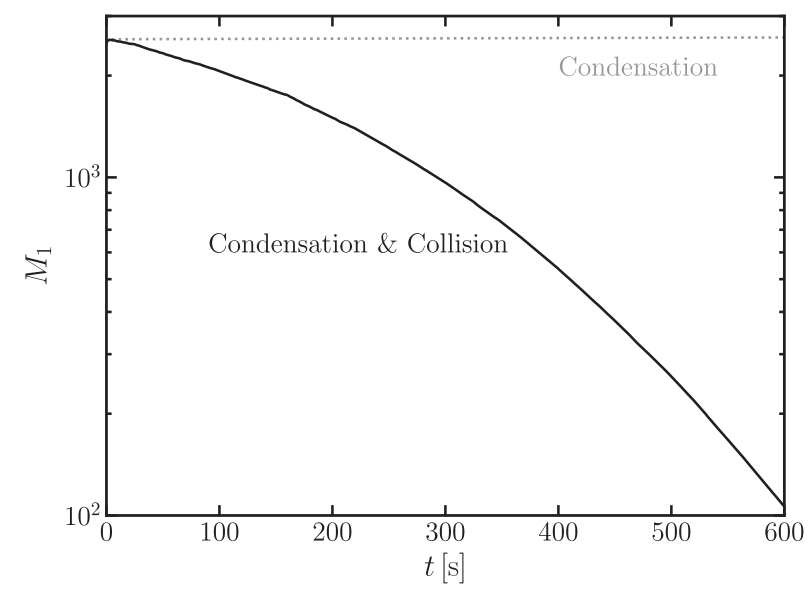

FIG. 2. Evolution of $M_{1}$ for simulations shown in Fig. 1.

The decreasing $C_{d, \text { rms }}(t)$ leads to a positive feedback on $T_{\mathrm{rms}}(t)$ and $s_{\mathrm{rms}}(t)$, and a negative feedback on $q_{v, \mathrm{rms}}(t)$. The buoyancy force $B$ is determined by temperature fluctuations $T^{\prime}$, water vapor mixing ratio fluctuations $q_{v}^{\prime}$, and the liquid-water mixing ratio $q_{l}$. The collisioncoalescence process leads to more intense local variations of $q_{l}$, which result in larger values of $q_{l, \mathrm{rms}}$. Therefore, $B_{\mathrm{rms}}(t)$ is enhanced by the collision-coalescence process through $q_{l}$. In our simulations, however, the enhanced $B$ does not affect the flow field since the random forcing overwhelms the buoyancy force in our simulations. Thus, collisional growth does not impact the condensational growth in the present DNS. This may change when the volume stirring of the flow is replaced by buoyant driving. Such driving could be more realistic, especially on larger scales that cannot be accessed in current state-of-the-art DNS.

Next, we investigate how condensational growth affects the collisional growth by comparing the time evolution of the droplet size distribution for three different cases: condensation only, collision only, and the combined process. Figure $3 \mathrm{a}$ shows the comparison of droplet size distributions when $\operatorname{Re}_{\lambda}=45$ and $\bar{\varepsilon}=0.039 \mathrm{~m}^{2} \mathrm{~s}^{-3}$. For the case with only condensation, the width of the droplet size distribution increases from a monodispersed initial distribution. When comparing the tail of the size distribution between the cases of collision only and that of the combined process, we see that the broadening from the condensational growth facilitates the collisional growth. The combined condensational and collisional growth leads to large tails of the size distribution. In Fig. 3b, we show the corresponding result for $\mathrm{Re}_{\lambda}=130$. At $t=600 \mathrm{~s}=10 \mathrm{~min}$, the radius of the droplet reaches about $400 \mu \mathrm{m}$, which is almost the size of falling raindrops. This time scale is close to the observed time scale for warm rain formation. It is worth noting that for the combined process, the droplet size distribution exhibits an obvious transition from condensational growth to collisional growth, as shown by the dip in the droplet size distribution. We recall that the radius of all droplets is initially $r_{\text {ini }} \equiv 10 \mu \mathrm{m}$. After the first collision, the droplet grows to twice the mass, giving a radius of $12.6 \mu \mathrm{m}$. Condensational growth leads to a few large droplets close from the initially monodispersed $10 \mu \mathrm{m}$ droplet distribution, which triggers the collision-coalescence process. For the case of $\operatorname{Re}_{\lambda}=130$ (cyan curves), the dips are less abrupt. This is due to the fact that larger value of $\operatorname{Re}_{\lambda}$ lead to stronger supersaturation fluctuations, which thus generate more large droplets.

To see how the tail of $f(r, t)$ depends on $\operatorname{Re}_{\lambda}$ for different configurations, we examine $a_{\zeta}$. As shown in Fig. 4, $a_{24}$ is insensitive to $\operatorname{Re}_{\lambda}$ when condensation is excluded, which is consistent with previous studies (Chen et al. 2018a; Li et al. 2018a). However, when condensation is included, $a_{24}$ increases with increasing $\mathrm{Re}_{\lambda}$. This demonstrates that the value of $\operatorname{Re}_{\lambda}$ affects collisional growth indirectly through condensation. For cases with only condensation, $a_{24}$ is larger for larger $\mathrm{Re}_{\lambda}$.

We have also investigated how different initial distributions affect the combined condensational and collisional growth. It is found that the condensation process makes the combined processes almost independent of the initial distribution; see the appendix for details.

The collisional growth of cloud droplets is very sensitive to the tails of droplet size distributions. A few large droplets can undergo a runaway collision-coalescence process by collecting small droplets. The cumulative collision time of these few large droplets is much shorter than the mean collision time (Kostinski and Shaw 2005). Thus, fluctuations play an important role in collisional growth. Condensational growth due to supersaturation fluctuations facilitates this runaway collision-coalescence process by generating the few large droplets as demonstrated in this study.

\section{b. Effect of turbulence on combined condensational and collisional growth}

To study the effect of turbulence on the combined condensational and collisional growth, we explore how the time evolution of the droplet size distributions depends on $\bar{\varepsilon}$ and $\mathrm{Re}_{\lambda}$ in the case when the growth of droplets is driven by both condensation and collisioncoalescence. Several previous works (Lanotte et al. 2009; Sardina et al. 2015; Siewert et al. 2017; Li et al. 2019) showed that condensational growth is enhanced with increasing $\operatorname{Re}_{\lambda}$, but is insensitive to $\bar{\varepsilon}$. Collisional growth, however, depends on $\bar{\varepsilon}$ and is insensitive to $\operatorname{Re}_{\lambda}$ (Ayala et al. 2008; Chen et al. 2016; Li et al. 2018a). Therefore, we expect that the combined condensational and collisional growth depends on both $\operatorname{Re}_{\lambda}$ and $\bar{\varepsilon}$. 

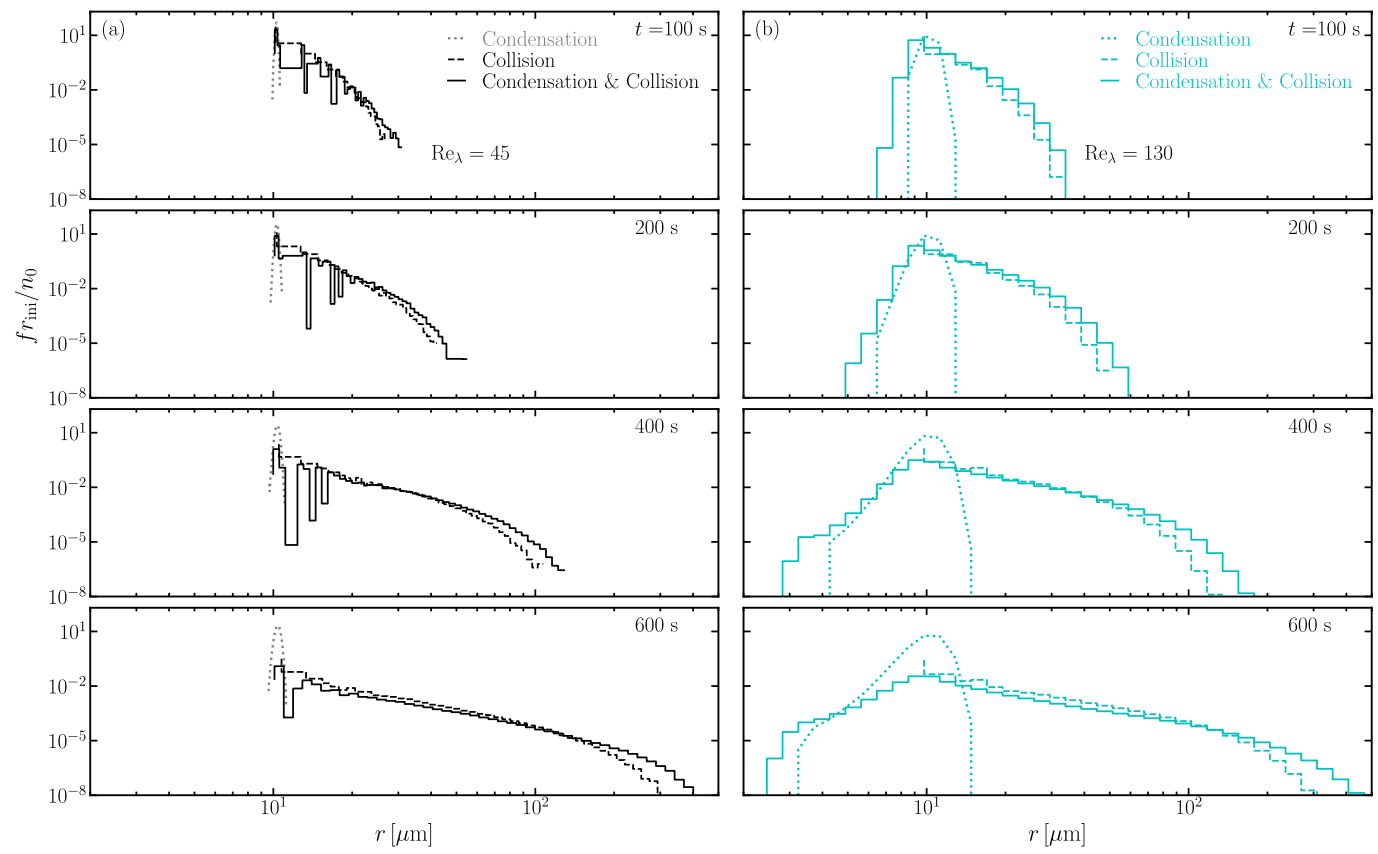

FIG. 3. Comparison of droplet size distributions for three cases: condensational growth (dotted lines; runs $\mathrm{C} 1$ and E1 in Table 1; Li et al. 2019), collisional growth (dashed lines; runs C2 and E2 in Table 1; Li et al. 2018a), and the combined processes (solid lines; runs $C$ and $E$ in Table 1). (a) $\operatorname{Re}_{\lambda}=45$ and (b) $\operatorname{Re}_{\lambda}=130$.

Let us first inspect how the evolution of $T, q_{v}, C_{d}$, and $s$ depends on $\operatorname{Re}_{\lambda}$ and $\bar{\varepsilon}$. Figure 5 shows that the rms values of these quantities increase as $\operatorname{Re}_{\lambda}$ increases, but they only depend weakly upon $\bar{\varepsilon}$ (cf. the blue, magenta, and black lines for runs $\mathrm{A}, \mathrm{B}$, and $\mathrm{C}$, respectively). This result is consistent with the conclusion of Sardina et al. (2015) and Li et al. (2019), where only the condensation/evaporation process was investigated, but now it is also verified for the combined condensational and collisional growth.

Figure 6a shows the time evolution of the corresponding droplet size distributions at an early stage of rain formation. Due to turbulence-induced supersaturation fluctuations, the width of $f(r, t)$ broadens to a certain value. The first peak at $r=10 \mu \mathrm{m}$ and its width are almost the same for different $\bar{\varepsilon}$ at different times. The distributions exhibit the same characteristics as those of the simulations without collisions in runs $\mathrm{C} 1$ and E1 shown in Fig. 3. We attribute this feature to the condensational growth and its weak dependency on $\bar{\varepsilon}$ (Sardina et al. 2015; Li et al. 2019). The tail of the droplet size distribution becomes wider with increasing $\bar{\varepsilon}$, which is attributed to the dependency of collisional growth on $\bar{\varepsilon}$. Figure $6 \mathrm{~b}$, on the other hand, shows the time evolution of the droplet size distributions for different $\operatorname{Re}_{\lambda}$ at fixed $\bar{\varepsilon}$. The first peak exhibits the same shape and dependency on $\operatorname{Re}_{\lambda}$ as those in Fig. 3 where collisions were not included. The distributions of small droplets become wider with increasing $\operatorname{Re}_{\lambda}$, which is due to the fact that both evaporation and condensation are enhanced with increasing $\mathrm{Re}_{\lambda}$. This also indicates the strong spatial inhomogeneity of the supersaturation field. The tail of the droplet size distribution broadens with increasing $\operatorname{Re}_{\lambda}$. This is attributed to the condensational growth and its induced collisions since collisional growth mainly depends on $\bar{\varepsilon}$.

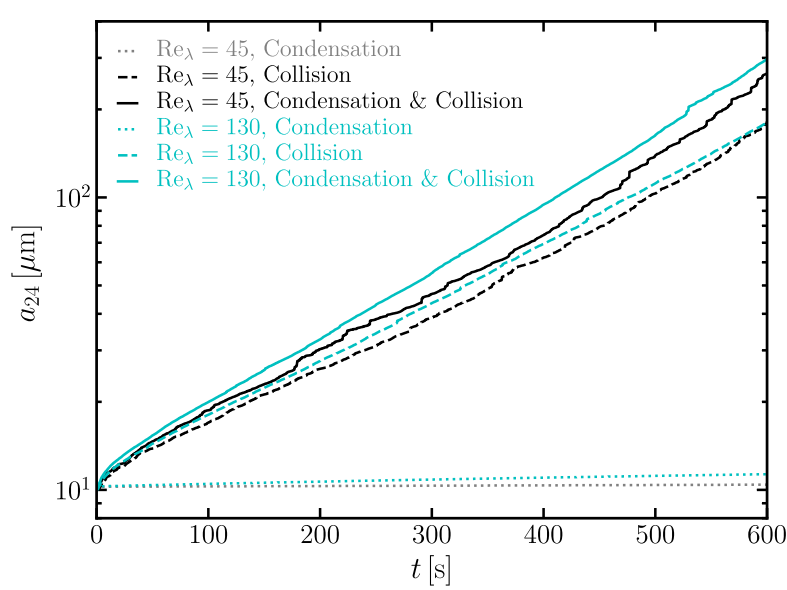

FIG. 4. Evolution of $a_{24}$ for different configurations: condensation only (dotted lines), collision-coalescence only (dashed lines), and condensation and collision-coalescence (solid lines). Black curves are for the cases with $\mathrm{Re}_{\lambda}=45$ and the cyan lines are for $\mathrm{Re}_{\lambda}=130$. 

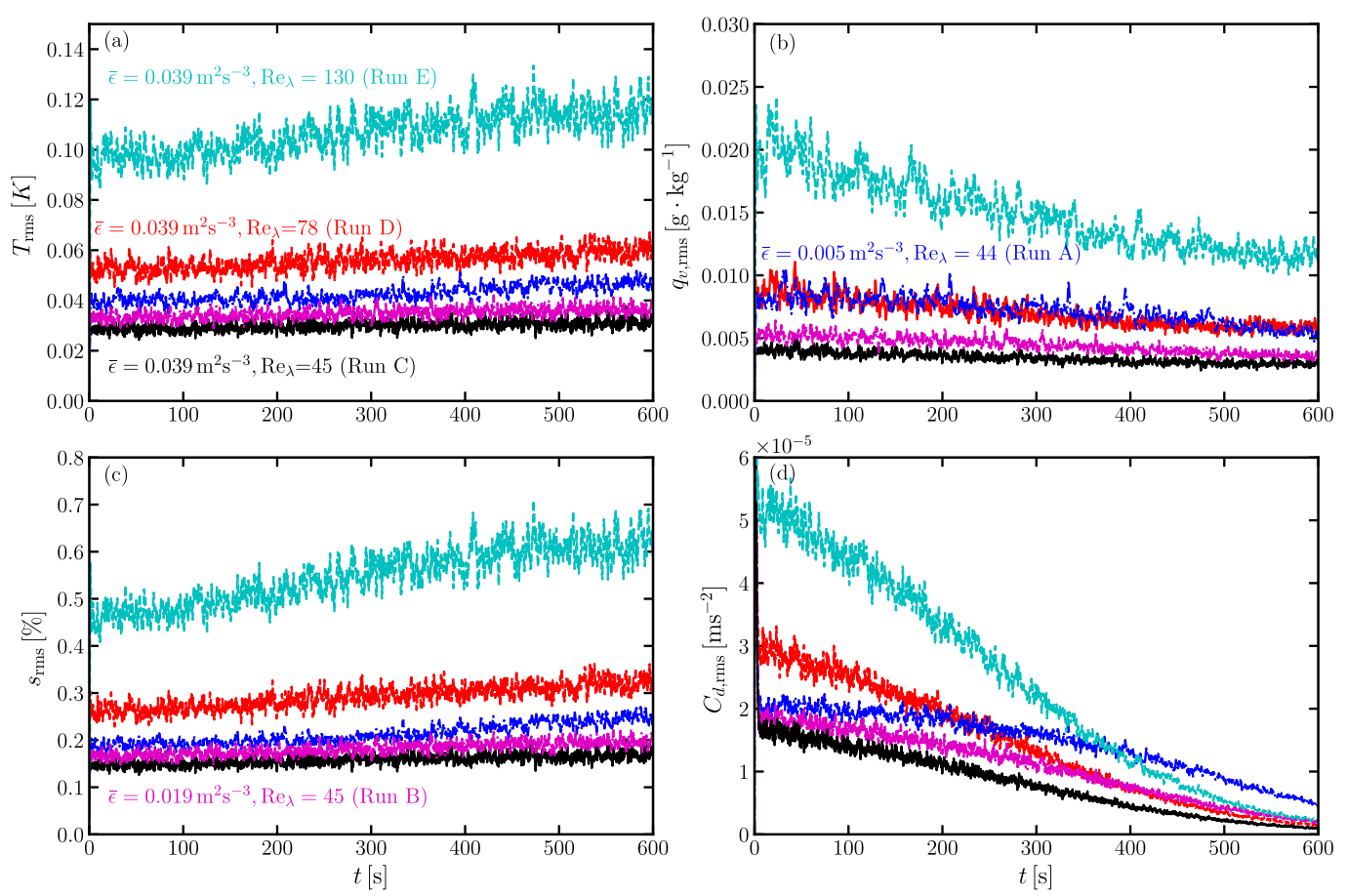

FIG. 5. Evolution of the rms values of (a) temperature, (b) water vapor mixing ratio, (c) supersaturation, and (d) condensation rate for runs A (blue), B (magenta), C (black), D (red), and E (cyan).

When the simulations ran for $600 \mathrm{~s}$, we observe that the $\mathrm{Re}_{\lambda}$ dependency becomes even stronger, as shown in Fig. 7. This is due to the fact that evaporation results in smaller droplets, as can be seen from the left tail of $f(r, t)$ in Fig. 7b. Larger values of $\mathrm{Re}_{\lambda}$ lead to stronger evaporation, and therefore the broadening effect due to condensation at the early stage of rain formation is counteracted by evaporation at the late stage. The probability density function (PDF) of $s$ broadens significantly with increasing values of $\mathrm{Re}_{\lambda}$. This implies that there is stronger evaporation (negative $s$ ) when $\mathrm{Re}_{\lambda}$ is larger; see Fig. 8. From 60 to $80 \mathrm{~s}$, the right tail of $f(r, t)$ due to condensation does not broaden. Instead, its left tail now extends further. The evolution of the dispersion of $f(r, t)$ is shown in Fig. 9, where we observe enhancement of $\sigma_{r} / \bar{r}$ with $\bar{\varepsilon}$ and $\mathrm{Re}_{\lambda}$. To characterize the tail of $f(r, t)$, we again inspect the higher moments of $f(r, t)$. As shown in Fig. 10, $a_{24}$ increases both with increasing $\bar{\varepsilon}$ (due to collision-coalescence) and with increasing $\operatorname{Re}_{\lambda}$ (due to condensation). Within the parameter ranges of $\bar{\varepsilon}$ and $\operatorname{Re}_{\lambda}$ in the present DNS, the $\bar{\varepsilon}$ dependency is more pronounced. We noticed that there is exactly one particle per superparticles for the smallest $f(r, t=600)$ for all the simulations. This excludes the possibility that the wider tail of $f(r, t)$ is due to a larger number of $N_{s}$ for this case with the largest $\mathrm{Re}_{\lambda}$. This is consistent with our statement in section $2 \mathrm{c}$ that $N_{p} / N_{\text {grid }}=0.1$ is adopted in all simulations to make sure that the tails of $f(r, t)$ are statistically converged.

\section{Discussion}

We have investigated how condensation and collisioncoalescence processes affect each other by comparing droplet size distributions for three cases: pure condensation, pure collision-coalescence, and the combined processes. We found that condensational growth broadens the droplet size distributions in the initial phase of droplet growth, after which collisional growth is triggered. The condensation-triggered collision is most pronounced for our largest Reynolds number, $\mathrm{Re}_{\lambda}=130$. In the present study, the collision-coalescence process only enhances the buoyancy force and affects the temperature, water vapor mixing ratio, and supersaturation slightly. Therefore, it does not influence the condensation process in the parameter range explored.

We have also studied the combined condensational and collisional growth at different $\bar{\varepsilon}$ and $\mathrm{Re}_{\lambda}$. We observed that the droplet size distribution broadens both with increasing $\bar{\varepsilon}$ and with increasing $\mathrm{Re}_{\lambda}$. The dependency on $\mathrm{Re}_{\lambda}$ can be explained as follows. Several previous DNS studies (Ayala et al. 2008; Chen et al. 2016; Li et al. 2018a) showed that collisional growth depends on $\bar{\varepsilon}$ and is insensitive to $\operatorname{Re}_{\lambda}$. The condensational growth, 

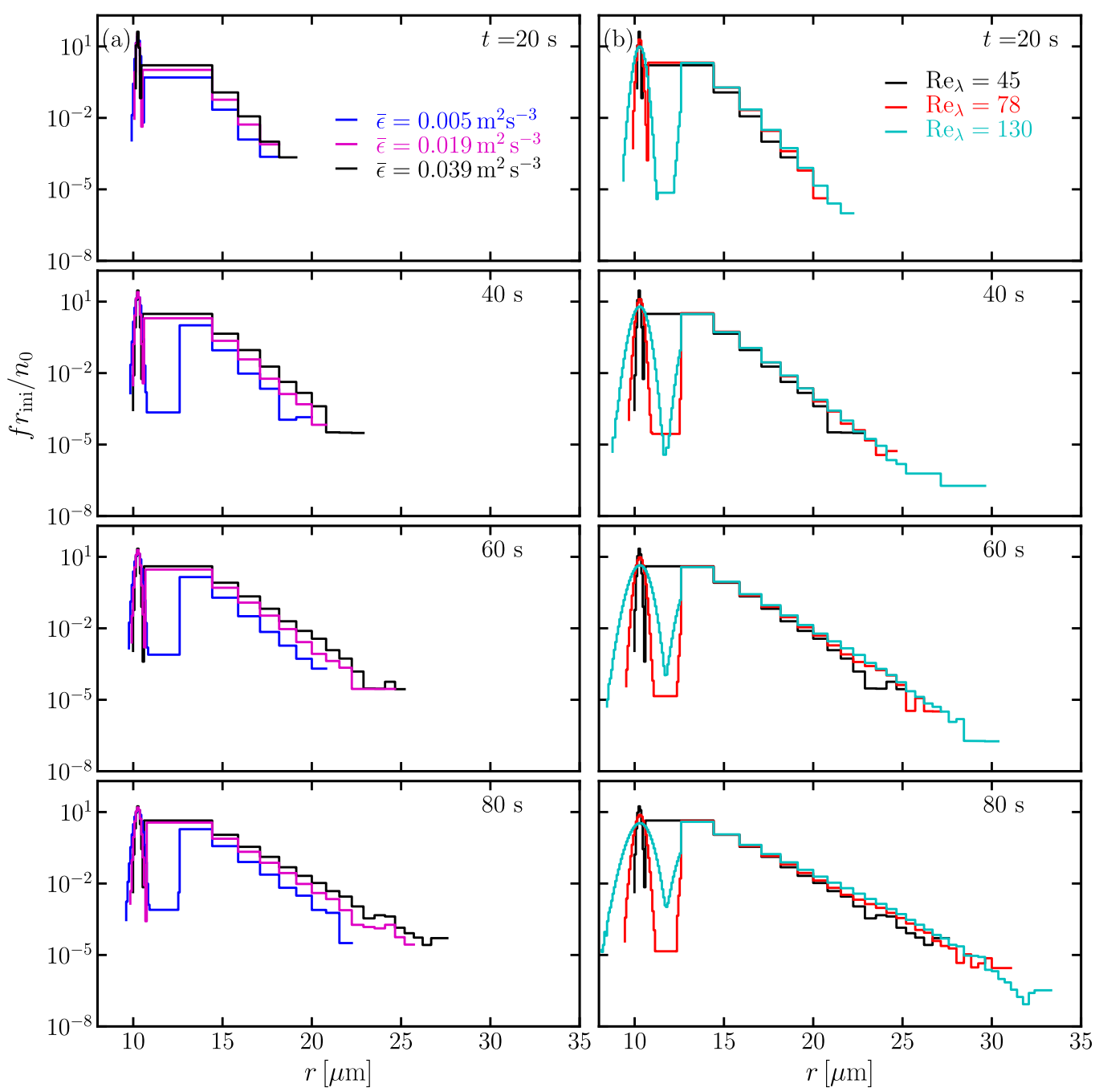

FIG. 6. Droplet size distributions for (a) different $\bar{\varepsilon}=0.005 \mathrm{~m}^{2} \mathrm{~s}^{-3}$ (blue solid lines), 0.019 (magenta solid lines), and 0.039 (black solid line) at fixed $\mathrm{Re}_{\lambda}=45$ (see runs A, B, and C in Table 1 for details) and (b) different $\operatorname{Re}_{\lambda}=45$ (black solid lines), 78 (red solid lines), and 130 (cyan solid line) at fixed $\bar{\varepsilon}=0.039 \mathrm{~m}^{2} \mathrm{~s}^{-3}$ (see runs C, D, and E in Table 1 for details).

instead, strongly depends on $\operatorname{Re}_{\lambda}$ and is insensitive to $\bar{\varepsilon}$ (Li et al. 2019). Also, in the present study, the comparison among cases of pure condensation, pure collision, and the combined process demonstrates that condensational growth triggers the collisional growth. Therefore, we conclude that the $\operatorname{Re}_{\lambda}$ dependency is caused by the condensation process, which indirectly enhances the collisional growth. The combined processes are also observed to depend on $\bar{\varepsilon}$, which is attributed to the dependency of the collisional growth on $\bar{\varepsilon}$. However, the largest local $\bar{\varepsilon}$ in warm clouds is about $\bar{\varepsilon}=10^{-1} \mathrm{~m}^{2} \mathrm{~s}^{-3}$ (Siebert et al. 2013), which is much smaller than the values achieved in the laboratory and engineering applications (Shaw 2003). Its effect on collisional growth should be treated with caution. The largest $\operatorname{Re}_{\lambda}$ is 130 and the lateral size of the domain is $L_{x}=0.5 \mathrm{~m}$ in the present DNS. In reality, we have $\operatorname{Re}_{\lambda} \approx 10^{4}$ (Siebert et al. 2006) in a cloud system with a typical turbulence integral length scale of $100 \mathrm{~m}$, which is two orders of magnitude larger than the $\operatorname{Re}_{\lambda}$ in the present DNS. It is expected that a higher $\operatorname{Re}_{\lambda}$ would lead to larger supersaturation fluctuations (Grabowski and Abade 2017), and therefore fast broadening of the size distribution, which facilitates the collisional growth. Our findings also support results of the laboratory experiment of Chandrakar et al. (2016) that supersaturation fluctuations are likely of leading importance for precipitation formation. Furthermore, we demonstrated numerically that supersaturation fluctuations enhance the collisional growth. 

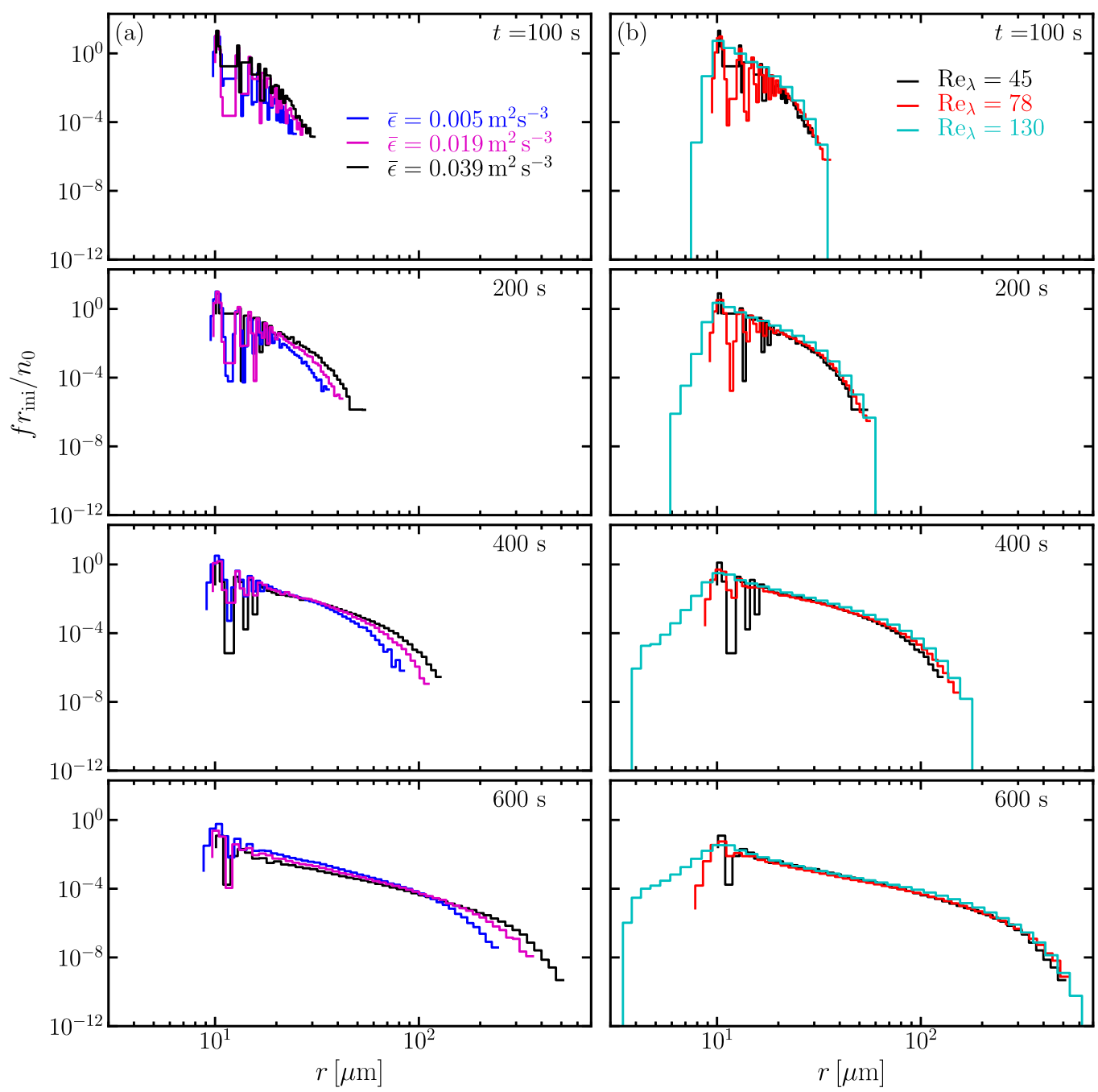

FIG. 7. As in Fig. 6, but at late times.

The classical treatment of condensational growth without turbulence, and with constant supersaturation results in a larger mean radius, but a narrower width of the size distribution. This reduces the relative velocity of potentially colliding pairs as they settle through the cloud. This implies slower collisional growth. Contrary to the classical treatment of condensational growth, our findings demonstrate that the supersaturation-fluctuation-induced condensational growth facilitates the collisional growth by broadening the width of the droplet size distribution.

Chen et al. (2018b) compared droplet size distributions for different $\bar{\varepsilon}$ when both condensation and collision were included. They attributed the condensation-induced collision to the fact that "condensational growth narrows the droplet size distribution (DSD) and provides a great number of similar-sized droplets" (Chen et al. 2018b), which is inconsistent with our finding that condensational growth produces wider distributions with increasing $\mathrm{Re}_{\lambda}$ and therefore facilitates the collisional growth. However, we emphasize that there are two crucial differences compared to our present model. First, the mean updraft cooling included by Chen et al. (2018b) may suppress the supersaturation-fluctuation-induced broadening of the droplet size distributions, first found by Sardina et al. (2018). Mean updraft cooling may result in more similar-sized droplets generated by the condensation process, which leads to their enhanced collision rate. Second, they included hydrodynamic interactions between droplets. This may modify the way how turbulence affects the collisional growth discussed here. In the present study, these two differences result in an overestimation of the combined collisional and condensational growth. When comparing the tail of $f(r, 400)$ in Fig. 7 with Fig. 1 of Chen et al. (2018b), our value is about $20 \%$ larger. 


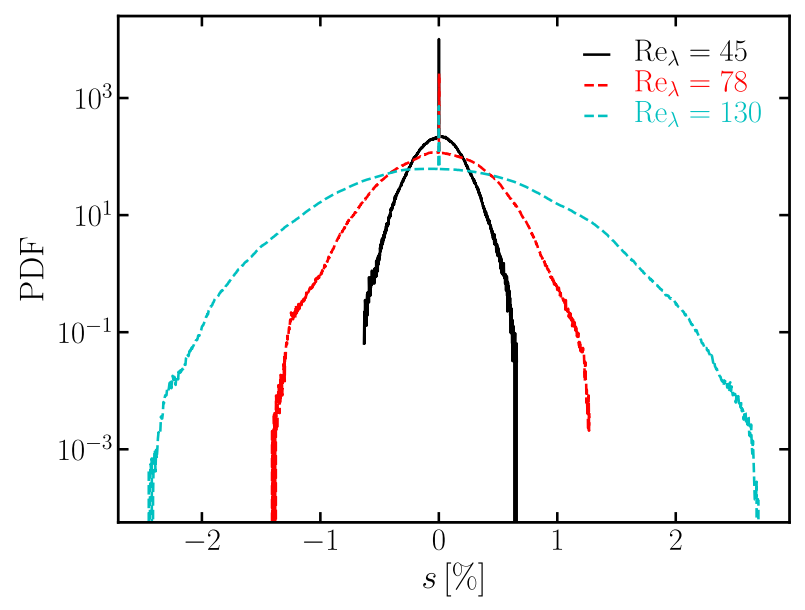

FIG. 8. PDF of $s$ for different $\operatorname{Re}_{\lambda}$ at $t=600 \mathrm{~s}$. Simulations are as in Fig. 7b.

In the present study, supersaturation fluctuations are caused purely by local condensation rate $C_{d}$ being affected by turbulence. This results in extreme supersaturation values, especially for the case of the largest $\mathrm{Re}_{\lambda}$, as shown by the tail of the PDF of $s$ in Fig. 8. No equilibrium state of the supersaturation field is obtained; see Fig. 5c. This is due to the following reason. In the case of pure condensation, supersaturation fluctuations become stationary as they relax to an equilibrium state (Sardina et al. 2015; $\mathrm{Li}$ et al. 2019). In the present study, the collision-coalescence process, however, leads to a continuous growth of droplet sizes (no droplet breakup is included), and alters the local concentration of droplets. This prevents supersaturation fluctuations reaching an equilibrium state.

The continuous evolution of droplet size distributions is sustained by supersaturation-fluctuation-induced broadening and the collision-coalescence process. In the pure condensation case, due to supersaturation fluctuations, the standard deviation of the droplet surface area is proportional to $\operatorname{Re}_{\lambda} t^{1 / 2}$ (Sardina et al. 2015; Li et al. 2019). This leads to continuous broadening of droplet size distributions in the absence of mean updraft cooling (Sardina et al. 2018). More importantly, the droplet size distribution is further broadened by the collisioncoalescence process. As discussed in section 2a, stochastic forcing is adopted in the present study, which cannot sufficiently capture large scales of turbulence. This is limited by the state-of-the-art supercomputer power. This is why all the DNS studies of the turbulence and cloud microphysics communities (e.g., Saito and Gotoh 2018; Chen et al. 2018b) have employed volume stirring.

Our study lends some support to the notion of "lucky" droplets (Kostinski and Shaw 2005), first proposed by Telford (1955). The lucky-droplet model assumes that there is a larger droplet among many small ones to initiate the runaway growth (Kostinski and Shaw 2005; Wilkinson 2016). The question is where the first few lucky droplets originate. Kostinski and Shaw (2005) proposed that the first few lucky droplets could be the result of giant condensation nuclei. The present study indicates that the first few lucky droplets could result from condensational growth driven by supersaturation fluctuations caused by turbulence.

\section{Conclusions}

We have found that the growth of cloud droplets in warm clouds is substantially affected by both the Reynolds number and the mean energy dissipation rate. The condensational growth is driven by supersaturation fluctuations. Supersaturation fluctuations are governed by fluctuations of temperature and the water vapor
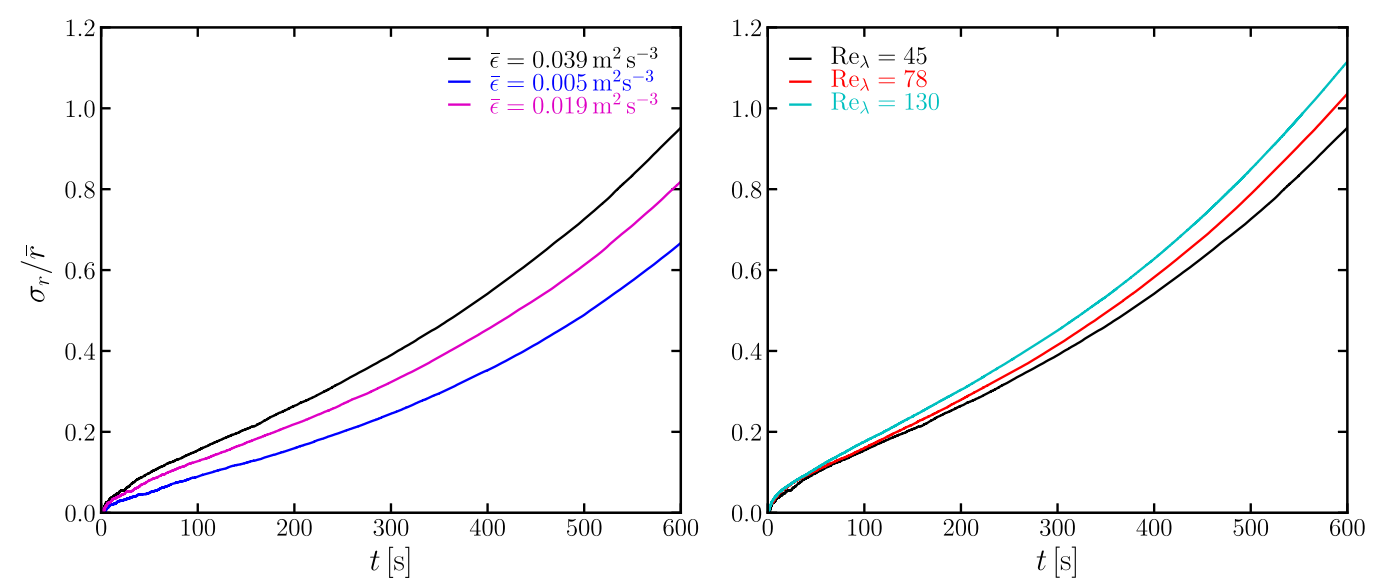

FIG. 9. Evolution of $\sigma_{r} / \bar{r}$ for the simulations shown in Fig. 7. 

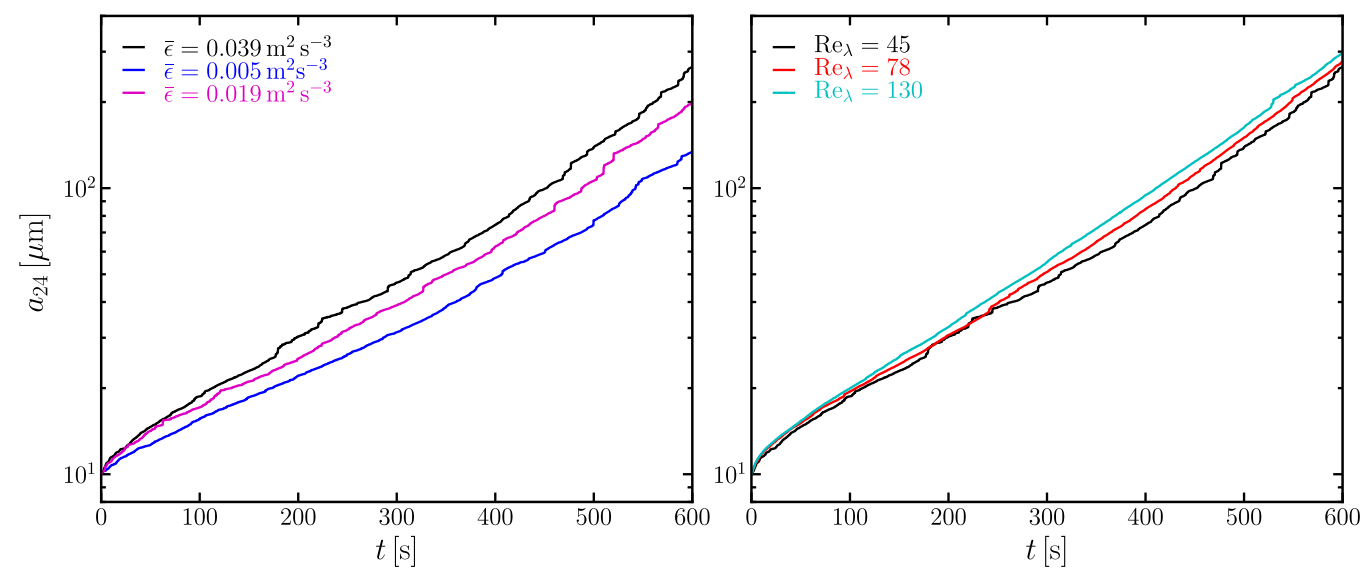

FIG. 10. Evolution of $a_{24}$ for the simulations shown in Fig. 7.

mixing ratio, which were found to increase with increasing Reynolds number (Lanotte et al. 2009; Sardina et al. 2015; Siewert et al. 2017; Li et al. 2019). This results in a broadening of droplet size distributions, which is contrary to the classical understanding of condensational growth in laminar flows that leads to a narrowing size distribution. When the droplet size distribution has reached a certain width, collisional growth starts to dominate. It is then affected by the mean energy-dissipation rate. In other words, the value of the Reynolds number influences the collisional growth indirectly through condensation. Therefore, the combined condensational and collisional growth is influenced by both the Reynolds number and the mean energy dissipation rate. With the limited Reynolds numbers and the relatively small domain size employed in the present DNS study, we observed how the broadening of droplet size distributions driven by supersaturation fluctuations facilitates the collisional growth at an early stage of rain formation. Evaporation becomes stronger with increasing Reynolds number, which counteracts the broadening of the droplet size distribution with increasing Reynolds number.

In the present study, the collision and coalescence efficiencies were assumed to be unity, which may substantially overestimate the collisional growth. For example, the largest particle Reynolds number is in excess of 500 in some of our DNS, resulting in droplet rebound or breakup, which can be accounted for in the coalescence efficiency (Lamb and Verlinde 2011, p. 406). This suggests the existence of an upper bound for the enhancement of turbulence on collisional growth. Since the turbulence-induced collision efficiency is a very challenging problem (Grabowski and Wang 2013), it may be useful to incorporate a robust scheme of collision efficiency in the superparticle approach. Entrainment is also omitted, which is supposed to cause strong supersaturation fluctuations. Aerosol activation is not included in the present study. Invoking all the cloud microphysical processes is computationally extremely demanding-even on modern supercomputers. We strive to achieve this in future studies.

Due to the aforementioned limitations, we have not attempted to compare droplet size distributions obtained from the current work with observational data. Such a step would make sense once we address the limitations mentioned above and have a more realistic representation of the large scales, where the flow is dominated by convective driving instead of volume stirring, as in the present work.

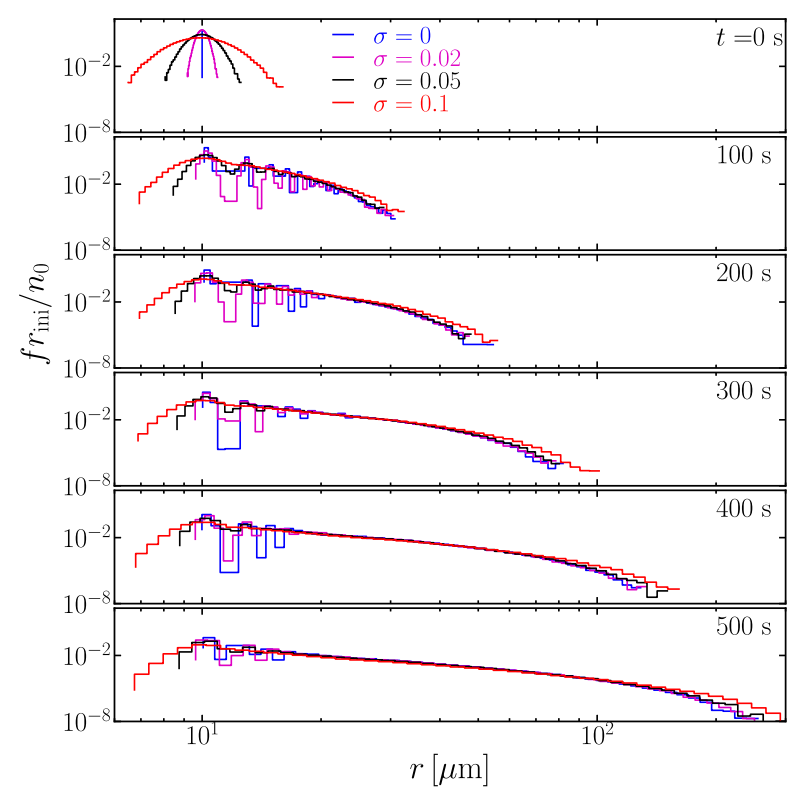

FIG. A1. Comparison of droplet size distributions for different width $\sigma_{\text {ini. }}$. See runs $\mathrm{C}$ in Table 1 for details of the numerical setup. 


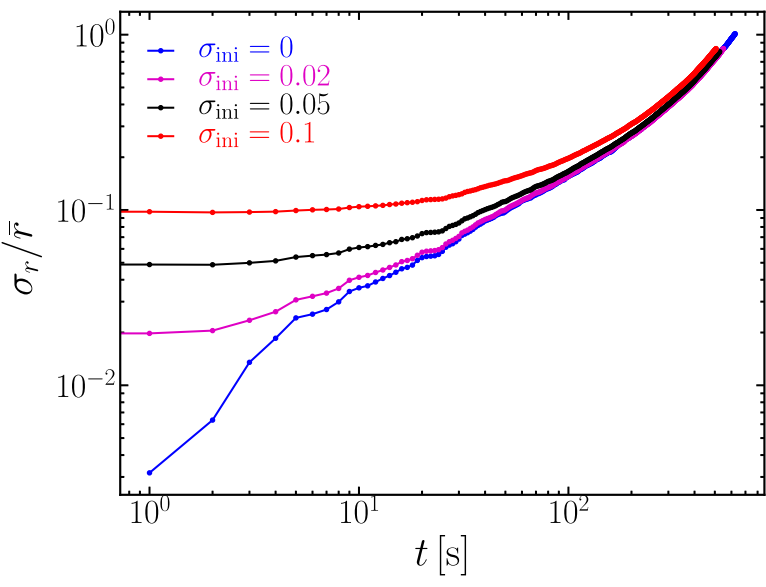

FIG. A2. The corresponding relative dispersion $\sigma_{r} / \bar{r}$ of droplet size distributions shown in Fig. A1.

Acknowledgments. This work was supported through the FRINATEK Grant 231444 under the Research Council of Norway, SeRC, the Israel Science Foundation governed by the Israel Academy of Sciences (Grant 1210/15), the University of Colorado through its support of the George Ellery Hale visiting faculty appointment, the grant "Bottlenecks for particle growth in turbulent aerosols" from the Knut and Alice Wallenberg Foundation, Dnr. KAW 2014.0048, and Vetenskapsrådet with Grant 2017-03865. N.E.L.H. acknowledges the Research project Gaspro, financed by the Research Council of Norway (267916). The simulations were performed using resources provided by the Swedish National Infrastructure for Computing (SNIC) at the Royal Institute of Technology in Stockholm and Chalmers Centre for Computational Science and Engineering (C3SE). This work also benefited from computer resources made available through the Norwegian NOTUR program, under Award NN9405K. The source code used for the simulations of this study, the Pencil code, is freely available online (https://github.com/pencil-code/).

\section{APPENDIX}

\section{Independence of the Initial Distribution}

In section $3 \mathrm{a}$, we investigated how different initial distributions affect the combined condensational and collisional growth. In this appendix, we give further details and show how $f(r, t)$ depends on $\sigma_{\text {ini. }}$. Figure A1 shows that $f(r, t)$ is insensitive to the width $\sigma_{\text {ini }}$ of the initial size distribution. As shown in Fig. A2, $\sigma_{r} / \bar{r}$ is insensitive to $\sigma_{\text {ini }}$ at late times. This is consistent with the behavior of $f(r, t)$ shown in Fig. A1. Since $\sigma_{r} / \bar{r}$ only involves the second moment of the radius $r$, it is not

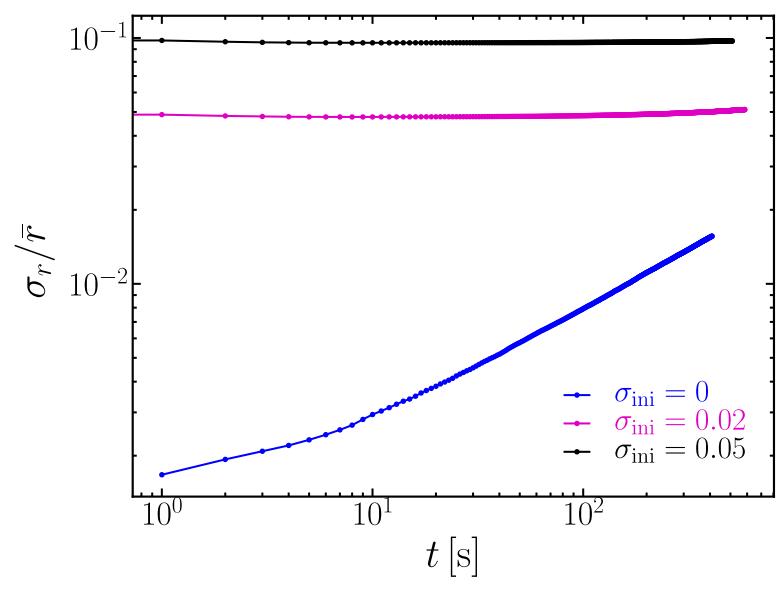

FIG. A3. The relative dispersion $\sigma_{r} / \bar{r}$ for different $\sigma_{\text {ini }}$, where the growth is solely driven by condensation.

able to characterize the tail of $f(r, t)$. Therefore, we use high moments of $r$ as defined in Eq. (10) of section 3. We show $\sigma_{r} / \bar{r}$ for comparison.

To investigate why $\sigma_{r} / \bar{r}$ is insensitive to $\sigma_{\text {ini }}$, we examine how the condensation process responds to $\sigma_{\text {ini }}$. It is evident that the condensation process is damped when $\sigma_{\text {ini }} \geq 0.02$, as shown in Fig. A3. This suggests that condensation makes the combined processes almost independent of the initial size distribution, which counteracts the initial width dependency of the collisioncoalescence process ( $\mathrm{Li}$ et al. 2018a).

\section{REFERENCES}

Abade, G. C., W. W. Grabowski, and H. Pawlowska, 2018: Broadening of cloud droplet spectra through eddy hopping: Turbulent entraining parcel simulations. J. Atmos. Sci., 75, 3365-3379, https://doi.org/10.1175/JAS-D-18-0078.1.

Ayala, O., B. Rosa, and L.-P. Wang, 2008: Effects of turbulence on the geometric collision rate of sedimenting droplets. Part 2. Theory and parameterization. New J. Phys., 10, 099802, https:// doi.org/10.1088/1367-2630/10/7/075016.

Bec, J., L. Biferale, M. Cencini, A. Lanotte, and F. Toschi, 2010: Intermittency in the velocity distribution of heavy particles in turbulence. J. Fluid Mech., 646, 527-536, https://doi.org/ 10.1017/S0022112010000029.

Berry, E. X., and R. L. Reinhardt, 1974: An analysis of cloud drop growth by collection: Part I. Double distributions. J. Atmos. Sci., 31, 1814-1824, https://doi.org/10.1175/1520-0469(1974) $031<1814: \mathrm{AAOCDG}>2.0 . \mathrm{CO} ; 2$.

Bhatnagar, A., K. Gustavsson, B. Mehlig, and D. Mitra, 2018a: Relative velocities in bi-disperse turbulent aerosols: Simulations and theory. arXiv, https://arxiv.org/abs/ 1809.10440.

,-- , and D. Mitra, 2018b: Statistics of the relative velocity of particles in turbulent flows: Monodisperse particles. Phys. Rev., 97E, 023105, https://doi.org/10.1103/PhysRevE.97.023105.

Chandrakar, K. K., W. Cantrell, K. Chang, D. Ciochetto, D. Niedermeier, M. Ovchinnikov, R. A. Shaw, and F. Yang, 2016: Aerosol indirect effect from turbulence-induced broadening of 
cloud-droplet size distributions. Proc. Natl. Acad. Sci. USA, 113, 14 243-14248, https://doi.org/10.1073/pnas.1612686113.

Chen, S., P. Bartello, M. K. Yau, P. A. Vaillancourt, and K. Zwijsen, 2016: Cloud droplet collisions in turbulent environment: Collision statistics and parameterization. J. Atmos. Sci., 73, 621-636, https://doi.org/10.1175/JAS-D-15-0203.1.

— , M. K. Yau, and P. Bartello, 2018a: Turbulence effects of collision efficiency and broadening of droplet size distribution in cumulus clouds. J. Atmos. Sci., 75, 203-217, https://doi.org/ 10.1175/JAS-D-17-0123.1.

,,--- , and L. Xue, 2018b: Bridging the condensationcollision size gap: A direct numerical simulation of continuous droplet growth in turbulent clouds. Atmos. Chem. Phys., 18, 7251-7262, https://doi.org/10.5194/acp-18-7251-2018.

Chun, J., D. L. Koch, S. L. Rani, A. Ahluwalia, and L. R. Collins, 2005: Clustering of aerosol particles in isotropic turbulence. J. Fluid Mech., 536, 219-251, https://doi.org/10.1017/ S0022112005004568.

Desai, N., S. Glienke, J. Fugal, and R. Shaw, 2019: Search for microphysical signatures of stochastic condensation in marine boundary layer clouds using airborne digital holography. J. Geophys. Res. Atmos., 124, 2739-2752, https://doi.org/ 10.1029/2018JD029033.

Devenish, B., and Coauthors, 2012: Droplet growth in warm turbulent clouds. Quart. J. Roy. Meteor. Soc., 138, 1401-1429, https://doi.org/10.1002/qj.1897.

Dziekan, P., and H. Pawlowska, 2017: Stochastic coalescence in Lagrangian cloud microphysics. Atmos. Chem. Phys., 17, 13 509-13 520, https://doi.org/10.5194/acp-17-13509-2017.

Falkovich, G., A. Fouxon, and G. Stepanov, 2002: Acceleration of rain initiation by cloud turbulence. Nature, 419, 151-154, https://doi.org/10.1038/nature00983.

Franklin, C. N., 2008: A warm rain microphysics parameterization that includes the effect of turbulence. J. Atmos. Sci., 65, 17951816, https://doi.org/10.1175/2007JAS2556.1.

_ , P. A. Vaillancourt, M. Yau, and P. Bartello, 2005: Collision rates of cloud droplets in turbulent flow. J. Atmos. Sci., 62, 2451-2466, https://doi.org/10.1175/JAS3493.1.

Götzfried, P., B. Kumar, R. A. Shaw, and J. Schumacher, 2017: Droplet dynamics and fine-scale structure in a shearless turbulent mixing layer with phase changes. J. Fluid Mech., 814, 452-483, https://doi.org/10.1017/jfm.2017.23.

Grabowski, W. W., and L.-P. Wang, 2013: Growth of cloud droplets in a turbulent environment. Annu. Rev. Fluid Mech., 45, 293 324, https://doi.org/10.1146/annurev-fluid-011212-140750.

—_, and G. C. Abade, 2017: Broadening of cloud droplet spectra through eddy hopping: Turbulent adiabatic parcel simulations. J. Atmos. Sci., 74, 1485-1493, https://doi.org/10.1175/ JAS-D-17-0043.1.

— , H. Morrison, S.-i. Shima, G. C. Abade, P. Dziekan, and H. Pawlowska, 2018: Modeling of cloud microphysics: Can we do better? Bull. Amer. Meteor. Soc., 100, 655-672, https:// doi.org/10.1175/BAMS-D-18-0005.1.

Grover, S., and H. Pruppacher, 1985: The effect of vertical turbulent fluctuations in the atmosphere on the collection of aerosol particles by cloud drops. J. Atmos. Sci., 42, 2305-2318, https://doi.org/ 10.1175/1520-0469(1985)042<2305:TEOVTF $>2.0 . C O ; 2$.

Gustavsson, K., and B. Mehlig, 2011: Distribution of relative velocities in turbulent aerosols. Phys. Rev., 84E, 045304, https:// doi.org/10.1103/PhysRevE.84.045304.

- , and - 2014: Relative velocities of inertial particles in turbulent aerosols. J. Turbul., 15, 34-69, https://doi.org/10.1080/ 14685248.2013.875188.
— heavy particles in turbulence. Adv. Phys., 65, 1-57, https:// doi.org/10.1080/00018732.2016.1164490.

— S. Vajedi, and B. Mehlig, 2014: Clustering of particles falling in a turbulent flow. Phys. Rev. Lett., 112, 214501, https:// doi.org/10.1103/PhysRevLett.112.214501.

Haugen, N. E. L., A. Brandenburg, and W. Dobler, 2004: Simulations of nonhelical hydromagnetic turbulence. Phys. Rev., 70E, 016308, https://doi.org/10.1103/PhysRevE.70.016308.

Igel, A. L., and S. C. van den Heever, 2017: The importance of the shape of cloud droplet size distributions in shallow cumulus clouds. Part II: Bulk microphysics simulations. J. Atmos. Sci., 74, 259-273, https://doi.org/10.1175/JAS-D-15-0383.1.

Johansen, A., and M. Lambrechts, 2017: Forming planets via pebble accretion. Annu. Rev. Earth Planet. Sci., 45, 359-387, https://doi.org/10.1146/annurev-earth-063016-020226.

_ A. N. Youdin, and Y. Lithwick, 2012: Adding particle collisions to the formation of asteroids and Kuiper belt objects via streaming instabilities. Astron. Astrophys., 537, A125, https:// doi.org/10.1051/0004-6361/201117701.

Jonas, P., 1996: Turbulence and cloud microphysics. Atmos. Res., 40, 283-306, https://doi.org/10.1016/0169-8095(95)00035-6.

Khain, A., M. Pinsky, T. Elperin, N. Kleeorin, I. Rogachevskii, and A. Kostinski, 2007: Critical comments to results of investigations of drop collisions in turbulent clouds. Atmos. Res., 86, 1-20, https://doi.org/10.1016/j.atmosres.2007.05.003.

Kostinski, A. B., and R. A. Shaw, 2005: Fluctuations and luck in droplet growth by coalescence. Bull. Amer. Meteor. Soc., 86, 235-244, https://doi.org/10.1175/BAMS-86-2-235.

Koziol, A. S., and H. Leighton, 1996: The effect of turbulence on the collision rates of small cloud drops. J. Atmos. Sci., 53, 1910-1920, https://doi.org/10.1175/1520-0469(1996)053<1910: TEOTOT $>2.0 . \mathrm{CO} ; 2$.

Kumar, B., J. Schumacher, and R. A. Shaw, 2014: Lagrangian mixing dynamics at the cloudy-clear air interface. J. Atmos. Sci., 71, 2564-2580, https://doi.org/10.1175/JAS-D-13-0294.1.

Lamb, D., and J. Verlinde, 2011: Physics and Chemistry of Clouds. Cambridge University Press, 600 pp.

Lanotte, A. S., A. Seminara, and F. Toschi, 2009: Cloud droplet growth by condensation in homogeneous isotropic turbulence. J. Atmos. Sci., 66, 1685-1697, https://doi.org/10.1175/2008JAS2864.1.

Lau, K., and H. Wu, 2003: Warm rain processes over tropical oceans and climate implications. Geophys. Res. Lett., 30, 2290, https://doi.org/10.1029/2003GL018567.

Li, X.-Y., A. Brandenburg, N. E. L. Haugen, and G. Svensson, 2017: Eulerian and Lagrangian approaches to multidimensional condensation and collection. J. Adv. Model. Earth Syst., 9, 1116-1137, https://doi.org/10.1002/2017MS000930.

,-- , G. Svensson, N. E. L. Haugen, B. Mehlig, and I. Rogachevskii, 2018a: Effect of turbulence on collisional growth of cloud droplets. J. Atmos. Sci., 75, 3469-3487, https:// doi.org/10.1175/JAS-D-18-0081.1.

_ B. Behlig, G. Svensson, A. Brandenburg, and N. E. Haugen, 2018b: Fluctuations and growth histories of cloud droplets: Superparticle simulations of the collision-coalescence process. arXiv, https://arxiv.org/abs/1810.07475.

—, G. Svensson, A. Brandenburg, and N. E. Haugen, 2019: Cloud-droplet growth due to supersaturation fluctuations in stratiform clouds. Atmos. Chem. Phys., 19, 639-648, https:// doi.org/10.5194/acp-19-639-2019.

Marchioli, C., and Coauthors, 2008: Statistics of particle dispersion in direct numerical simulations of wall-bounded turbulence: Results of an international collaborative benchmark test. 
Int. J. Multiphase Flow, 34, 879-893, https://doi.org/10.1016/ j.ijmultiphaseflow.2008.01.009.

Mehaddi, R., F. Candelier, and B. Mehlig, 2018: Inertial drag on a sphere settling in a stratified fluid. arXiv, https://arxiv.org/abs/ 1802.10416.

Meibohm, J., L. Pistone, K. Gustavsson, and B. Mehlig, 2017: Relative velocities in bidisperse turbulent suspensions. Phys. Rev., 96E, 061102, https://doi.org/10.1103/PhysRevE.96.061102.

Onishi, R., and A. Seifert, 2016: Reynolds-number dependence of turbulence enhancement on collision growth. Atmos. Chem. Phys., 16, $12441-12455$, https://doi.org/10.5194/ acp-16-12441-2016.

—_, K. Matsuda, and K. Takahashi, 2015: Lagrangian tracking simulation of droplet growth in turbulence-turbulence enhancement of autoconversion rate. J. Atmos. Sci., 72, 25912607, https://doi.org/10.1175/JAS-D-14-0292.1.

Paoli, R., and K. Shariff, 2009: Turbulent condensation of droplets: Direct simulation and a stochastic model. J. Atmos. Sci., 66, 723-740, https://doi.org/10.1175/2008JAS2734.1.

Pinsky, M., and A. Khain, 2004: Collisions of small drops in a turbulent flow. Part II: Effects of flow accelerations. J. Atmos. Sci., 61, 1926-1939, https://doi.org/10.1175/1520-0469(2004) $061<1926$ :COSDIA $>2.0 . \mathrm{CO} ; 2$.

__ _ _ , and M. Shapiro, 2007: Collisions of cloud droplets in a turbulent flow. Part IV: Droplet hydrodynamic interaction. J. Atmos. Sci., 64, 2462-2482, https://doi.org/ 10.1175/JAS3952.1.

, - — , and H. Krugliak, 2008: Collisions of cloud droplets in a turbulent flow. Part V: Application of detailed tables of turbulent collision rate enhancement to simulation of droplet spectra evolution. J. Atmos. Sci., 65, 357-374, https://doi.org/ 10.1175/2007JAS2358.1.

Pruppacher, H. R., and J. D. Klett, 2012: Microphysics of Clouds and Precipitation. Springer Science and Business Media, $714 \mathrm{pp}$.

Reuter, G., R. De Villiers, and Y. Yavin, 1988: The collection kernel for two falling cloud drops subjected to random perturbations in a turbulent air flow: A stochastic model. J. Atmos. Sci., 45, 765-773, https://doi.org/10.1175/1520-0469(1988)045<0765: TCKFTF $>2.0 . \mathrm{CO} ; 2$

Rosa, B., H. Parishani, O. Ayala, W. W. Grabowski, and L.-P. Wang, 2013: Kinematic and dynamic collision statistics of cloud droplets from high-resolution simulations. New J. Phys., 15, 045032, https://doi.org/10.1088/1367-2630/15/4/ 045032 .

Saffman, P. G., and J. S. Turner, 1956: On the collision of drops in turbulent clouds. J. Fluid Mech., 1, 16-30, https://doi.org/ 10.1017/S0022112056000020.

Saito, I., and T. Gotoh, 2018: Turbulence and cloud droplets in cumulus clouds. New J. Phys., 20, 023001, https://doi.org/10.1088/ 1367-2630/aaa229.

Salazar, J. P., J. De Jong, L. Cao, S. H. Woodward, H. Meng, and L. R. Collins, 2008: Experimental and numerical investigation of inertial particle clustering in isotropic turbulence. J. Fluid Mech., 600, 245-256, https://doi.org/10.1017/ S0022112008000372.

Sardina, G., F. Picano, L. Brandt, and R. Caballero, 2015: Continuous growth of droplet size variance due to condensation in turbulent clouds. Phys. Rev. Lett., 115, 184501, https://doi.org/ 10.1103/PhysRevLett.115.184501.

, S. Poulain, L. Brandt, and R. Caballero, 2018: Broadening of cloud droplet size spectra by stochastic condensation: Effects of mean updraft velocity and CCN activation. J. Atmos. Sci., 75, 451-467, https://doi.org/10.1175/JAS-D-17-0241.1.
Schiller, L., and A. Naumann, 1933: Fundamental calculations in gravitational processing. Z. Ver. Dtsch. Ing., 77, 318-320.

Shaw, R. A., 2003: Particle-turbulence interactions in atmospheric clouds. Annu. Rev. Fluid Mech., 35, 183-227, https://doi.org/ 10.1146/annurev.fluid.35.101101.161125.

Shima, S., K. Kusano, A. Kawano, T. Sugiyama, and S. Kawahara, 2009: The super-droplet method for the numerical simulation of clouds and precipitation: A particle-based and probabilistic microphysics model coupled with a non-hydrostatic model. Quart. J. Roy. Meteor. Soc., 135, 1307-1320, https://doi.org/10.1002/qj.441.

Siebert, H., and R. A. Shaw, 2017: Supersaturation fluctuations during the early stage of cumulus formation. J. Atmos. Sci., 74, 975-988, https://doi.org/10.1175/JAS-D-16-0115.1.

_ K. Kehmann, and M. Wendisch, 2006: Observations of smallscale turbulence and energy dissipation rates in the cloudy boundary layer. J. Atmos. Sci., 63, 1451-1466, https://doi.org/ 10.1175/JAS3687.1.

, and Coauthors, 2013: The fine-scale structure of the trade wind cumuli over Barbados-An introduction to the CARRIBA project. Atmos. Chem. Phys., 13, 10 061-10 077, https://doi.org/ 10.5194/acp-13-10061-2013.

Siewert, C., J. Bec, and G. Krstulovic, 2017: Statistical steady state in turbulent droplet condensation. J. Fluid Mech., 810, 254280, https://doi.org/10.1017/jfm.2016.712.

Smoluchowsky, M., 1917: Mathematical theory of the kinetics of the coagulation of colloidal solutions. Z. Phys. C, 19, 129-135.

Srivastava, R., 1989: Growth of cloud drops by condensation: A criticism of currently accepted theory and a new approach. J. Atmos. Sci., 46, 869-887, https://doi.org/10.1175/1520-0469(1989) 046<0869:GOCDBC $>2.0 . \mathrm{CO} ; 2$.

Stephens, G. L., and J. M. Haynes, 2007: Near global observations of the warm rain coalescence process. Geophys. Res. Lett., $\mathbf{3 4}$ L20805, https://doi.org/10.1029/2007GL030259.

Sundaram, S., and L. R. Collins, 1997: Collision statistics in an isotropic particle-laden turbulent suspension. Part 1. Direct numerical simulations. J. Fluid Mech., 335, 75-109, https:// doi.org/10.1017/S0022112096004454.

Telford, J. W., 1955: A new aspect of coalescence theory. J. Meteor., 12, 436-444, https://doi.org/10.1175/1520-0469(1955)012<0436: ANAOCT $>2.0 . \mathrm{CO} ; 2$.

Unterstrasser, S., F. Hoffmann, and M. Lerch, 2017: Collection/ aggregation algorithms in Lagrangian cloud microphysical models: Rigorous evaluation in box model simulations. Geosci. Model Dev., 10, 1521-1548, https://doi.org/10.5194/ gmd-10-1521-2017.

Vaillancourt, P., M. K. Yau, and W. W. Grabowski, 2001: Microscopic approach to cloud droplet growth by condensation. Part I: Model description and results without turbulence. J. Atmos. Sci., 58, 1945-1964, https://doi.org/10.1175/1520-0469(2001) 058<1945:MATCDG >2.0.CO;2.

,,-- P. Bartello, and W. W. Grabowski, 2002: Microscopic approach to cloud droplet growth by condensation. Part II: Turbulence, clustering, and condensational growth. J. Atmos. Sci., 59, 3421-3435, https://doi.org/10.1175/1520-0469(2002) 059<3421:MATCDG $>2.0 . \mathrm{CO} ; 2$.

Wang, L.-P., and W. W. Grabowski, 2009: The role of air turbulence in warm rain initiation. Atmos. Sci. Lett., 10, 1-8, https:// doi.org/10.1002/asl.210.

Wilkinson, M., 2016: Large deviation analysis of rapid onset of rain showers. Phys. Rev. Lett., 116, 018501, https://doi.org/10.1103/ PhysRevLett.116.018501.

- and B. Mehlig, 2005: Caustics in turbulent aerosols. Europhys. Lett., 71, 186-192, https://doi.org/10.1209/ep1/i2004-10532-7. 
- - - and V. Bezuglyy, 2006: Caustic activation of rain showers. Phys. Rev. Lett., 97, 048501, https:/doi.org/10.1103/ PhysRevLett.97.048501.

Woittiez, E. J., H. J. Jonker, and L. M. Portela, 2009: On the combined effects of turbulence and gravity on droplet collisions in clouds: A numerical study. J. Atmos. Sci., 66, 19261943, https://doi.org/10.1175/2005JAS2669.1.

Xue, Y., L.-P. Wang, and W. W. Grabowski, 2008: Growth of cloud droplets by turbulent collision-coalescence. J. Atmos. Sci., 65, 331-356, https://doi.org/10.1175/2007JAS2406.1.
Yang, F., P. Kollias, R. A. Shaw, and A. M. Vogelmann, 2018: Cloud droplet size distribution broadening during diffusional growth: Ripening amplified by deactivation and reactivation. Atmos. Chem. Phys., 18, 7313-7328, https://doi.org/10.5194/acp-18-7313-2018.

Yau, M. K., and R. Rogers, 1996: A Short Course in Cloud Physics. Elsevier, 304 pp.

Zsom, A., and C. P. Dullemond, 2008: A representative particle approach to coagulation and fragmentation of dust aggregates and fluid droplets. Astron. Astrophys., 489, 931-941, https:// doi.org/10.1051/0004-6361:200809921. 